\title{
On the AU Microscopii debris disk
}

\section{Density profiles, grain properties, and dust dynamics ${ }^{\star}$}

\author{
J.-C. Augereau ${ }^{1,2}$ and H. Beust ${ }^{1}$ \\ 1 Laboratoire d'Astrophysique de l'Observatoire de Grenoble, BP 53, 38041 Grenoble Cedex 9, France \\ e-mail: augereau@obs.ujf-grenoble.fr \\ 2 Leiden Observatory, PO Box 9513, 2300 RA Leiden, The Netherlands
}

Received 23 September 2005 / Accepted 15 April 2006

\section{ABSTRACT}

\begin{abstract}
Context. AU Mic is a young M-type star surrounded by an edge-on optically thin debris disk that shares many common observational properties with the disk around $\beta$ Pictoris. In particular, the scattered light surface brightness profile falls off as $\sim r^{-5}$ outside $120 \mathrm{AU}$ for $\beta$ Pictoris and $35 \mathrm{AU}$ for $\mathrm{AU}$ Mic. In both cases, the disk color rises as the distance increases beyond these reference radii.

Aims. In this paper, we present the first comprehensive analysis of the AU Mic disk properties since the system was resolved by Kalas et al. (2004, Science, 303, 1990). We explore whether the dynamical model, which successfully reproduces the $\beta$ Pictoris brightness profile (e.g., Augereau et al. 2001, A\&A, 370, 447), could apply to AU Mic.

Methods. We calculate the surface density profile of the AU Mic disk by performing the inversion of the near-IR and visible scattered light brightness profiles measured by Liu (2004, Science, 305, 1442) and Krist et al. (2005, AJ, 129, 1008), respectively. We discuss the grain properties by analysing the blue color of the disk in the visible (Krist et al.2005) and by fitting the disk spectral energy distribution. Finally, we evaluate the radiation and wind forces on the grains. The impact of the recurrent X-ray and UV-flares on the dust dynamics is also discussed.

Results. We show that irrespective of the mean scattering asymmetry factor of the grains, most of the emission arises from an asymmetric, collisionally-dominated region that peaks close to the surface brightness break around $35 \mathrm{AU}$. The elementary scatterers at visible wavelengths are found to be sub-micronic, but the inferred size distribution underestimates the number of large grains, resulting in sub-millimeter emissions that are too low compared to the observations. From our inversion procedure, we find that the $V$ to $H$-band scattering cross sections ratio increases outside $40 \mathrm{AU}$, in line with the observed color gradient of the disk. This behavior is expected if the grains have not been produced locally, but placed in orbits of high eccentricity by a size-dependent pressure force, resulting in a paucity of large grains beyond the outer edge of the parent bodies' disk. Because of the low luminosity of AU Mic, radiation pressure is inefficient to diffuse the smallest grains in the outer disk, even when the flares are taken into account. Conversely, we show that a standard, solar-like stellar wind generates a pressure force onto the dust particles that behaves much like a radiation pressure force. With an assumed $\dot{M} \simeq 3 \times 10^{2} \dot{M}_{\odot}$, the wind pressure overcomes the radiation pressure, and this effect is enhanced by the stellar flares. This greatly contributes to populating the extended AU Mic debris disk and explains the similarity between the $\beta$ Pictoris and AU Mic brightness profiles. In both cases, the color gradient beyond $120 \mathrm{AU}$ for $\beta$ Pictoris and $35 \mathrm{AU}$ for AU Mic, is believed to be a direct consequence of the dust dynamics.
\end{abstract}

Key words. stars: circumstellar matter - stars: individual: AU Mic - stars: flare - planetary systems: formation - scattering

\section{Introduction}

Recent visible and near-infrared coronagraphic imaging of the nearby M-type star AU Mic revealed its geometrically and optically thin circumstellar dust disk (Kalas et al. 2004; Liu 2004; Krist et al. 2005; Metchev et al. 2005; Fitzgerald et al. 2004). The disk, seen almost perfectly edge-on, extends over several hundreds of AU diameter. The disk morphology presents interesting similarities with the well-studied disk around the young A5V main sequence star $\beta$ Pictoris: both disks are seen edgeon, imaging has evidenced radial and vertical asymmetries, and, importantly, the radial surface brightness profiles have similar shapes. Both dust disks are furthermore thought to be made of short-lived collisional debris and the gas to be largely dissipated (Liu et al. 2004; Brandeker et al. 2004; Roberge et al. 2005). AU Mic and $\beta$ Pictoris would, moreover, be co-eval since they would belong to the same nearby stellar association aged $12_{-4}^{+8}$ Myr (Barrado y Navascués et al. 1999; Zuckerman et al. 2001).

* Appendices A-C are only available in electronic form at http://www.edpsciences.org
Do these similarities between the two debris disks point toward similar ongoing dynamical processes? The vertical asymmetries of the $\beta$ Pictoris disk seen in scattered light have been modeled assuming the presence of a planet on an inclined orbit with respect to the disk midplane (Mouillet et al. 1997; Augereau et al. 2001). The AU Mic disk appears slightly vertically distorted within a $50 \mathrm{AU}$ radius, but current observations do not readily show large-scale vertical asymmetries similar to those observed in the $\beta$ Pictoris disk. Hypothetic planetary companion(s) around AU Mic should therefore be on low inclination orbit(s) with respect to the disk midplane. The AU Mic disk also shows small-scale structures appearing as local brightness enhancements not present on each side of the disk (Liu 2004; Krist et al. 2005). Such small-scale side-to-side asymmetries have also been reported for $\beta$ Pictoris (Pantin et al. 1997; Telesco et al. 2005 ) and could correspond to local enhancements of the collision rate due to planetesimals trapped with a planet (e.g., Roques et al. 1994; Telesco et al. 2005).

More intriguing is the similarity between the midplane surface brightness profiles of the AUMic and $\beta$ Pictoris disks in scattered light. The observed profiles both decrease almost 
linearly with increasing the distance from the star, then drop with power law indexes between -4.4 and -5.5 . The break between the two power-law regimes is observed around $35 \mathrm{AU}$ for $\mathrm{AU}$ Mic and $120 \mathrm{AU}$ for $\beta$ Pictoris. According to Augereau et al. (2001), the surface brightness break around $120 \mathrm{AU}$ in the $\beta$ Pictoris disk corresponds to the outer edge of the collisional planetesimal ring that produces the observed dust particles. The stellar radiation pressure of the central A5V star places the smallest bound dust particles on orbits of high eccentricity with the same periastron as their parent bodies. These grains spend a large fraction of their time close to apoastron, distributing in this way the dust particles over a wide range of distances far from the place they have been produced. This results in a disk that extends over hundreds of AU with a generic scattered light surface brightness profile proportional to $r^{-5}$ beyond the break at $120 \mathrm{AU}$, as was observed (Lecavelier des Étangs et al. 1996; Augereau et al. 2001; Thébault \& Augereau 2005). Because of the drag that the gas can induce on the dust particles, this model requires a low gas density to keep the scattered light profile consistent with the observations. Thébault \& Augereau (2005) use this effect to place an upper limit on the gas density in the $\beta$ Pictoris disk.

The previous model, which successfully explains the scattered light brightness profile of the $\beta$ Pictoris disk, does not a priori apply to the AU Mic disk because of the low stellar luminosity of the central M-type star. The stellar radiation pressure on the grains is expected to be small, and, according to Kalas et al. (2004), the steep slope of the scattered light profile outside $35 \mathrm{AU}$ would then reflect the profile of the parent bodies' distribution rather than the effect of the radiation pressure, as is the case for $\beta$ Pictoris. In this scenario, the similarities between the AU Mic and $\beta$ Pictoris surface brightness profiles would just be coincidental. This conclusion nevertheless depends on uncertain parameters. Radiation pressure depends on the grains properties (composition, size, etc.), which are poorly constrained. AU Mic is also a flare star with UV and X-ray rises that could periodically increase the radiation pressure force on the grains. Alternatively, a hypothetical stellar wind could create a corpuscular pressure force that would help diffusing the dust particles at large distances. Finally, the collisional activity of the disk has not been precisely determined and requires the disk surface density to be properly estimated.

In this paper, we invert HST visible and ground-based nearinfrared surface brightness observations to calculate surface density profiles. We derive the integral equation that links the observed profiles to the physical quantities we want to recover in Sect. 2, and in Sect. 3 we detail our numerical approach. We then discuss the shape of the disk surface density as a function of the anisotropic scattering properties of the grains (Sect. 3.4). In Sect. 4, we constrain the grain size distribution based on the blue color of the disk in the visible (Krist et al. 2005), and we derive some disk properties (mass and optical thickness). Finally, we argue in Sects. 5 and 6 that the observed $r^{-5}$ surface brightness profile outside $35 \mathrm{AU}$ in scattered light is not coincidental, but instead reflects, like in the $\beta$ Pictoris disk, the ongoing dynamics of the observed dust grains. Our scenario involves both the observed stellar flares and the presence of a wind drag force.

\section{Surface brightness profile}

\subsection{Notations and assumptions}

We note $(x, y, z)$, the observer's line of sight Cartesian frame with the star at the origin and with the $x$-axis pointing to the

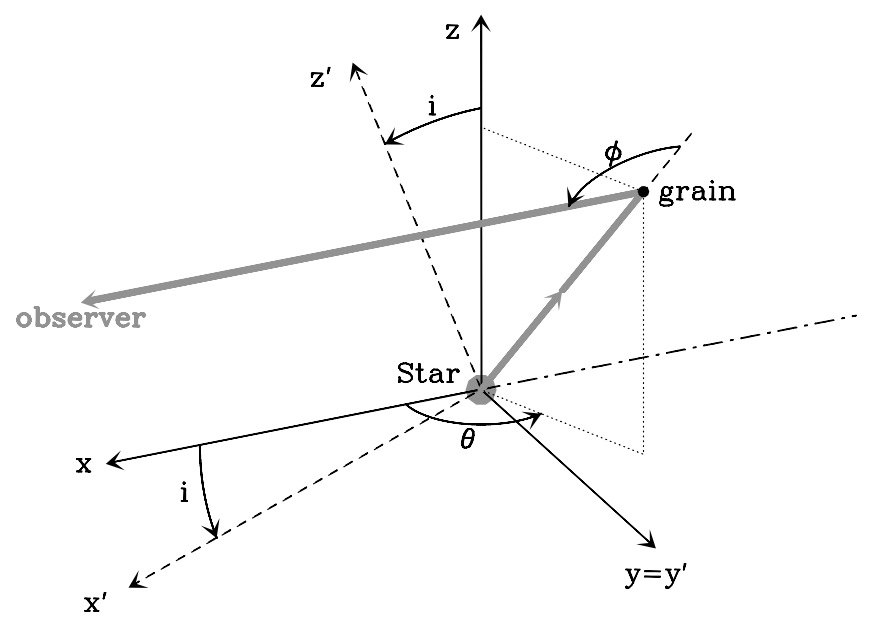

Fig. 1. Notations used in this study (see Sect. 2.1).

observer (Fig. 1). We consider a disk rotated around the $y$-axis at an angle $i$ with $i=0$ corresponding to an edge-on disk. We assume $-\pi / 2<i<\pi / 2$ to have $\cos i>0$. We note $\left(x^{\prime}, y^{\prime}, z^{\prime}\right)$, the Cartesian frame attached to the disk with the star at the origin and with the $z^{\prime}$-axis perpendicular to the disk midplane. We let $y^{\prime}=y$, which implies that the $y^{\prime}$-axis corresponds to the apparent major axis of the disk when $i \neq 0$. We furthermore define $(r, \theta, z)$ and $\left(r^{\prime}, \theta^{\prime}, z^{\prime}\right)$, the cylindrical coordinates in the line of sight and disk frames, respectively. We let $r_{\max }^{\prime}$ be the maximal radius of the disk, and $d_{\text {star }}$ the stellar heliocentric distance.

The AU Mic disk is assumed to be optically thin in all directions and at all wavelengths, which implies that the grains are directly illuminated by the central star. This assumption is justified by the low fractional dust disk luminosity $\left(L_{\text {disk }} / L_{*} \simeq 6 \times 10^{-4}\right.$, Liu et al. 2004), the low extinction of the star, and the lack of a central dark lane along the disk midplane in the visible and at near-IR wavelengths, as has been regularly observed for optically thick edge-on disks.

To account for light anisotropic scattering by the grains, we introduce the scattering angle $\phi$ with $\phi=0$ corresponding to forward scattering (see Fig. 1). Since $r_{\max }^{\prime} \ll d_{\text {star }}$, we will assume in the following that $\theta=-\phi$ for the grains with $z=0$.

\subsection{Scattered light surface brightness profile}

The surface brightness of an optically thin disk measured at a projected distance $y$ from the star along the $y$-axis (i.e., at $z=0$ ) is written

$B(y)=\int_{-\infty}^{+\infty} \rho\left(r^{\prime}, \theta^{\prime}, z^{\prime}\right) \Phi(r, \phi) \mathrm{d} x$,

where $\rho\left(r^{\prime}, \theta^{\prime}, z^{\prime}\right)$ is the disk number density and $\Phi(r, \phi)$ is the total flux measured at Earth and emitted by a grain at position $(r, \theta=-\phi, z=0)$. This flux is a function of the observing wavelength, but to simplify the notation, we will not indicate this dependence in the equations.

At the distances probed by the visible and near-IR coronagraphic images, the thermal emission from the grains contributes little to the disk brightness, and in the following, we will only consider the single scattering of the stellar light by the dust particles. $\Phi(r, \phi)$ then reads $\Phi^{*} \sigma_{\text {sca }} r^{-2} f(\phi)$, where $\Phi^{*}$ is the stellar flux at Earth at the considered wavelength, and $\sigma_{\text {sca }}$ and $f(\phi)$ are, respectively, the scattering cross section and the scattering phase function of the grains averaged over the grain size distribution. Here, we implicitly assumed that the line supporting any incident 
stellar ray is an axis of symmetry for the light scattered by the grains (phase function depending only on $\phi$ ). This is achieved, for instance, when the grains are spherical and homogeneous, and incoming light is unpolarized.

We assume that the disk is symmetric about the midplane and the $y$-axis. Equation (1) then can be re-written

$$
\begin{aligned}
B(y) & =\Phi^{*} \int_{0}^{+\infty} \sigma_{\mathrm{sca}} \rho\left(r^{\prime}, \theta^{\prime}, z^{\prime}\right) \frac{f(\phi)+f(\pi-\phi)}{r^{2}} \mathrm{~d} x \\
& =\Phi^{*} \int_{y}^{+\infty} \sigma_{\mathrm{sca}} \rho\left(r^{\prime}, \theta^{\prime}, z^{\prime}\right) \frac{f(\phi)+f(\pi-\phi)}{r^{2}} \frac{\partial x}{\partial r^{\prime}} \mathrm{d} r^{\prime} .
\end{aligned}
$$

The infinite bound on $r^{\prime}$ in Eq. (3) can be replaced with the maximal disk radius $r_{\text {max }}^{\prime}$, since the dust density is assumed to be null beyond $r_{\text {max }}^{\prime}$. One can show that $\partial x / \partial r^{\prime}=r^{\prime} \cos ^{-2} i / \sqrt{r^{2}-y^{2}}$ for $0 \leq \phi \leq \pi / 2$ with $\sqrt{r^{2}-y^{2}}=\sqrt{r^{\prime 2}-y^{2}} / \cos i$. Moreover, $r^{2}=\left(r^{\prime 2}-y^{2} \sin ^{2} i\right) / \cos ^{2} i$ when $z=0$. The scattered light surface brightness of the disk at a distance $y$ from the star along the $y$-axis thus is

$$
\begin{aligned}
B(y)= & \Phi^{*} \int_{y}^{r_{\max }^{\prime}} \sigma_{\mathrm{sca}} \rho\left(r^{\prime}, \theta^{\prime}, z^{\prime}\right) \\
& \times \frac{f(\phi)+f(\pi-\phi)}{r^{\prime 2}-y^{2} \sin ^{2} i} \frac{\cos i}{\sqrt{r^{\prime 2}-y^{2}}} r^{\prime} \mathrm{d} r^{\prime} .
\end{aligned}
$$

In the previous equation, we left the scattering cross section under the integral sign since the dust properties (composition and/or size distribution) could, in principle, depend on the distance from the star. The grain size distribution in the $\beta$ Pictoris disk is, for instance, expected to be distance-dependent (Fig. 4 in Augereau et al. 2001), and the recent observations by Golimowski et al. (2006) support this model. The situation might be similar in the case of AU Mic, as suggested by the observed gradual change of disk color in scattered light along the disk midplane (Krist et al. 2005; Metchev et al. 2005, see also Sect. 4.1)

\subsection{The case of edge-on geometrically thin disks}

The AU Mic disk is seen edge-on, which simplifies Eq. (4) ( $i=0, r=r^{\prime}, \theta=\theta^{\prime}=-\phi$, and $z=z^{\prime}=0$ ). The brightness profiles shown in Fig. 2 have been obtained by integrating the disk surface brightness over a few pixels in the direction orthogonal to the disk midplane. Therefore, $B(y)$ as defined by Eq. (4), does not directly compare with the measured profile, and the integration over $z$ has to be taken into account.

We can take advantage of the integration over $z$ to get an estimate of the surface number density of the disk instead of the number density in the disk midplane. The grains that contribute to $S(y)$ in geometrically thin disks have their height $z$ above the disk midplane that obey $z^{2} \ll r^{2}$, which implies $\phi \simeq-\theta$. Because of the symmetries of the phase function, we, in addition, have $f(-\theta)=f(\theta)$ and $f(\pi+\theta)=f(\pi-\theta)$ (see also Eq. (8)). We approximate the scattered light surface brightness profile measured along the disk midplane of an edge-on disk and integrated over $\Delta z / 2 \ll r$ in a direction perpendicular to the midplane by

$S(y) \simeq \Phi^{*} \int_{y}^{r_{\max }}(f(\theta)+f(\pi-\theta)) \frac{\sigma_{\mathrm{sca}} \Sigma(r, \theta)}{r \sqrt{r^{2}-y^{2}} D_{z}(r, \theta)} \mathrm{d} r$,

with $\Sigma(r, \theta)=\int_{-\infty}^{+\infty} \rho(r, \theta, z) \mathrm{d} z$

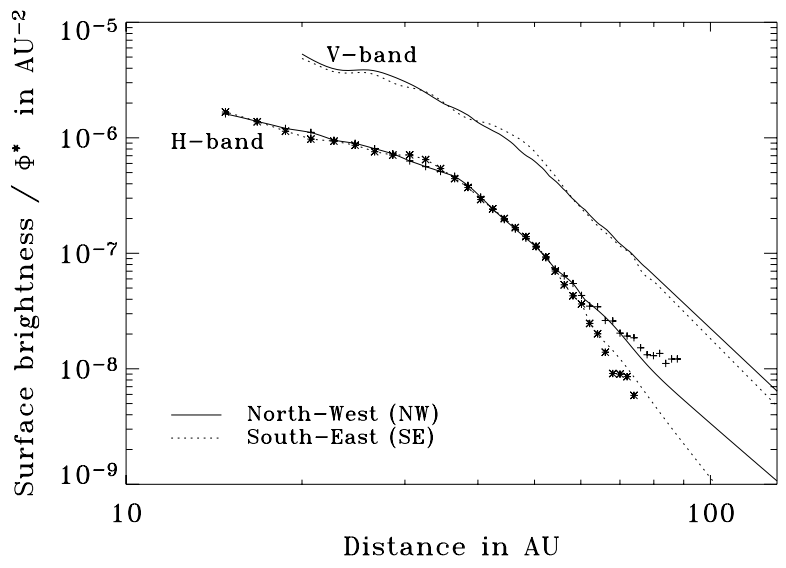

Fig. 2. Scattered light surface brightness profiles of the AU Mic disk $S(y)$ (divided by the stellar flux $\Phi^{*}$ to allow for comparison) in the $H$-band (Liu 2004) and in the ACS $V$-band (Krist et al. 2005). Beyond $r=65 \mathrm{AU}$, the original $H$-band profiles were extrapolated with radial power laws (NW: $r^{-4.3}, \mathrm{SE}: r^{-6.1}$ ). The visible profiles were similarly extrapolated beyond $75 \mathrm{AU}$ (NW: $\left.r^{-4.7}, \mathrm{SE}: r^{-5.1}\right)$. The vertical shift between the two profiles has two origins: the disk color in scattered light (Sect. 4.1), and the difference in spatial extent $(\Delta z)$ in a direction orthogonal to the disk midplane used to measure the disk brightness.

and $D_{z}(r, \theta)=\Delta z \frac{\Sigma(r, \theta)}{\int_{-\Delta z / 2}^{+\Delta z / 2} \rho(r, \theta, z) \mathrm{d} z}$,

where $\Sigma(r, \theta)$ is the disk surface number density. Equation (5) makes the link between the observed brightness profile $S(y)$ and the disk properties (surface number density, phase function and scattering cross section). It depends, through $D_{z}(r, \theta)$, on the vertical height above the disk midplane over which the brightness profile has been integrated. Equation (5) belongs to the class of first-kind Volterra integral equations, but because of the singularity in $r=y$, it actually belongs to the sub-class of Abel integral equations.

\section{Inversion of the AU Mic brightness profiles}

To infer the surface density profile of the AU Mic disk from observed scattered light profiles, we adopt a direct approach that consists of performing a basic numerical inversion of Eq. (5). This presents several advantages over a classical fitting approach. In particular, this limits the number of free parameters, since the inversion does not depend on any parametrization of the surface density profiles. We can therefore efficiently explore the coupling between the surface density and the anisotropic scattering properties of the grains.

\subsection{Observed surface brightness profiles}

In this study, we consider two sets of scattered light brightness profiles $(S(y))$, both displayed in Fig. 2 . The first set of profiles was measured by Liu (2004) in the $H$-band $\left(\lambda_{\mathrm{c}}=1.63 \mu \mathrm{m}\right)$ at the Keck II 10-m telescope equipped with an adaptive optics system. A spatial resolution of $0.04^{\prime \prime}$, or $0.4 \mathrm{AU}$ at the distance of AU Mic $(9.94 \pm 0.13 \mathrm{pc})$, has been achieved and the profiles have been derived from an aperture $\Delta z=0.6^{\prime \prime}$ ( $\sim 6 \mathrm{AU}$ ) wide in the direction perpendicular to the disk midplane. The Keck $H$ band coronagraphic images probe the disk down to $\sim 15 \mathrm{AU}$. The second set of scattered light brightness profiles was obtained in the visible with the HST/ACS instrument by Krist et al. (2005). The ACS images have a resolution of $\sim 0.63 \mathrm{AU}$ and have been 
obtained with $\Delta z=0.25^{\prime \prime}(\sim 2.5 \mathrm{AU})$. The HST/ACS images present several features at distances closer than about $20 \mathrm{AU}$ that are suspect according to Krist et al. (2005). The published ACS surface brightness profiles were multiplied by a factor of 1.125 to account for some confusion regarding the relation to the ACS geometric distortion and the photometric calibration points assumed by the HST Synphot package (Krist 2006, private communication).

The HST/ACS visible and Keck-AO near-IR images of the AU Mic disk show some differences discussed in Krist et al. (2005). Their impact on the surface density profiles calculated with our inversion technique are addressed in Sect. 3.4. All observed brightness profiles were oversampled to reach a common resolution ( $h$ in Appendix A) of $0.2 \mathrm{AU}$. The observed profiles were furthermore extrapolated with radial power laws beyond $65 \mathrm{AU}$ for the $H$-band profiles and beyond $75 \mathrm{AU}$ for the visible profiles (Fig. 2). The profiles in Fig. 2 were obtained assuming $\Phi^{*}=12.7 \mathrm{Jy}$ in the $H$-band and $\Phi^{*}=1.27 \mathrm{Jy}$ in the HST/ACS $V$-band $(F 606 W)$.

\subsection{Scattering phase function}

The scattering phase function of solid particles can be very complex depending on their shape and composition. To efficiently explore the impact of the anisotropic scattering properties of the grains (term $f(\theta)+f(\pi-\theta)$ in Eq. (5)) on the surface density profiles calculated with the inversion procedure, we adopt the single parameter Henyey \& Greenstein (1941) phase function

$$
f(\phi)=\frac{1-g^{2}}{4 \pi\left(1+g^{2}-2 g \cos \phi\right)^{3 / 2}},
$$

where the parameter $g=\iint_{4 \pi} f(\phi) \cos \phi \mathrm{d} \Omega$ is referred to as the mean asymmetry factor $(g=0$ for isotropic scattering and $|g|=1$ for purely forward/backward scattering). The $g$ parameter is related to the scattering properties of the individual grains through the following relation that ensures that the total scattered flux is preserved

$$
\begin{aligned}
& g=\int_{s_{\min }}^{s_{\max }} \pi s^{2} Q_{\text {sca }} \times g \mathrm{~d} n(s) / \sigma_{\text {sca }} \\
& \text { with } \sigma_{\text {sca }}=\int_{s_{\min }}^{s_{\max }} \pi s^{2} Q_{\text {sca }} \mathrm{d} n(s),
\end{aligned}
$$

where $s$ is the grain size, $s_{\min }$ and $s_{\max }$ are the minimum and maximum grain sizes, respectively, and $\mathrm{d} n(s)$ is the unity normalized differential grain size distribution $\left(\int_{s_{\min }}^{s_{\max }} \mathrm{d} n(s)=1\right)$. $Q_{\text {sca }}$ is the dimensionless scattering efficiency, and $g$ is the asymmetry factor that depends on the grain properties (size, composition, structure, etc.) and on the wavelength.

To efficiently explore the impact of scattering anisotropy on the results and to limit the number of free parameters, we will assume that the $g$ parameter is a constant. The inversion algorithm can nevertheless account for mean asymmetry parameters that depend on the grain position in the disk. We will also only consider the absolute value of $g$ since we cannot distinguish between positive and negative values, due to the edge-on orientation. The asymmetry factors $\left|g^{H}\right|$ and $\left|g^{V}\right|$ in the $H$-band and ACS-V-band, respectively, are thus the only free physical parameters when reconstructing surface density profiles from scattered light profiles using Eq. (5).

\subsection{Vertical and azimuthal profiles}

Following Krist et al. (2005), we assume that the vertical profile of the disk density can be approximated by a Lorentzian function $\left(1+z^{2} / H^{2}(r)\right)^{-1}$. The scale height $H(r)$ is parametrized as follows: $H(20 \mathrm{AU})=0.8675 \mathrm{AU}, H(r) \propto r^{0.08}$ for $r \leq 49 \mathrm{AU}$, $H(r) \propto r^{2.5}$ for $49<r \leq 80 \mathrm{AU}$, and $H(r) \propto r^{0.3}$ for $r>80 \mathrm{AU}$. $D_{z}(r, \theta)$ thus writes

$D_{z}(r, \theta)=\Delta z \frac{\pi}{2} \arctan ^{-1}\left(\frac{\Delta z}{2 H(r)}\right)$,

such that $D_{z}(r, \theta)=\Delta z$ for $\Delta z \gg 2 H(r)$.

The scattered light images of the AU Mic disk show side-toside asymmetries. Also, to appreciate how the inversion of the NW brightness profile affects the inversion of the SE brightness profile (and reciprocally), we introduce an ad hoc dependence on $\theta$ in the inversion process, and we proceed to the inversion of the NW and SE brightness profiles simultaneously. We re-write the dust surface number density as $\Sigma(r, \theta)=\Sigma_{0}(r) \Theta(r, \theta)$, where $\Theta(r, \theta)$ is a unit-less function given in Appendix B and characterized by a single, purely geometrical, parameter $\delta$. The role of the $\Theta(r, \theta)$ function is to smoothly link the NW and SE sides of the disk. During the inversion process, the surface density is calculated approximately along the $y$-axis (see Fig. 1). The $\Theta(r, \theta)$ function forces the NW and SE surface density profiles to be identical along the $x$-axis and equal to their mean value.

\subsection{Surface density profiles}

To reconstruct the surface density profiles of the disk, we used a classical numerical scheme to invert Eq. (5). The algorithm is detailed in Appendix A for the case of edge-on disks, but it can easily be extended to the case of inclined disks (e.g., the ring about the F5 star HD 181327, Schneider et al. 2006). As a sanity check, the calculated surface density profiles were systematically incorporated into our 3D debris disk model to compute synthetic brightness profiles (Augereau et al. 1999, 2001). These synthetic profiles were always found to be in excellent agreement with the original observed profiles.

The results of our inversion procedure are reported in Fig. 3. This figure displays the various surface density profiles found, assuming four different values for the mean asymmetry parameter $|g|$. For each $|g|$ value, the top panel shows the surface density profile that reproduces the $H$-band scattered light surface brightness profile measured by Liu (2004), while the profile in the bottom panel is derived from the ACS-V-band observations (Krist et al. 2005). The surface density profiles are found to depend quite sensibly on the assumed $|g|$ value. Large $|g|$ values promote the emission at scattering angles close to 0 or $\pi$ (depending on the sign of $g$ ), which is achieved when the grains are at distances from the star that are large, compared to their projected distance ( $r \gg y$ in Eq. (5)). The amount of dust at large distances thus tends to increase with increasing $|g|$ values, and the regions interior to the disk therefore become more and more dust-depleted. As a result, the disk derived from the $H$-band brightness profiles resembles a ring peaked around $35 \mathrm{AU}$ for sufficiently large $\left|g^{H}\right|$ values. Furthermore, $|g|$ cannot be significantly larger than $\sim 0.6$, since for larger $|g|$ values, the inversion algorithm requires the surface density profiles to be negative at some radii to compensate for the too large flux, compared to the observations, intercepted on the line of sight.

There are obvious differences between the surface density profiles obtained from the two data sets used in this study. 

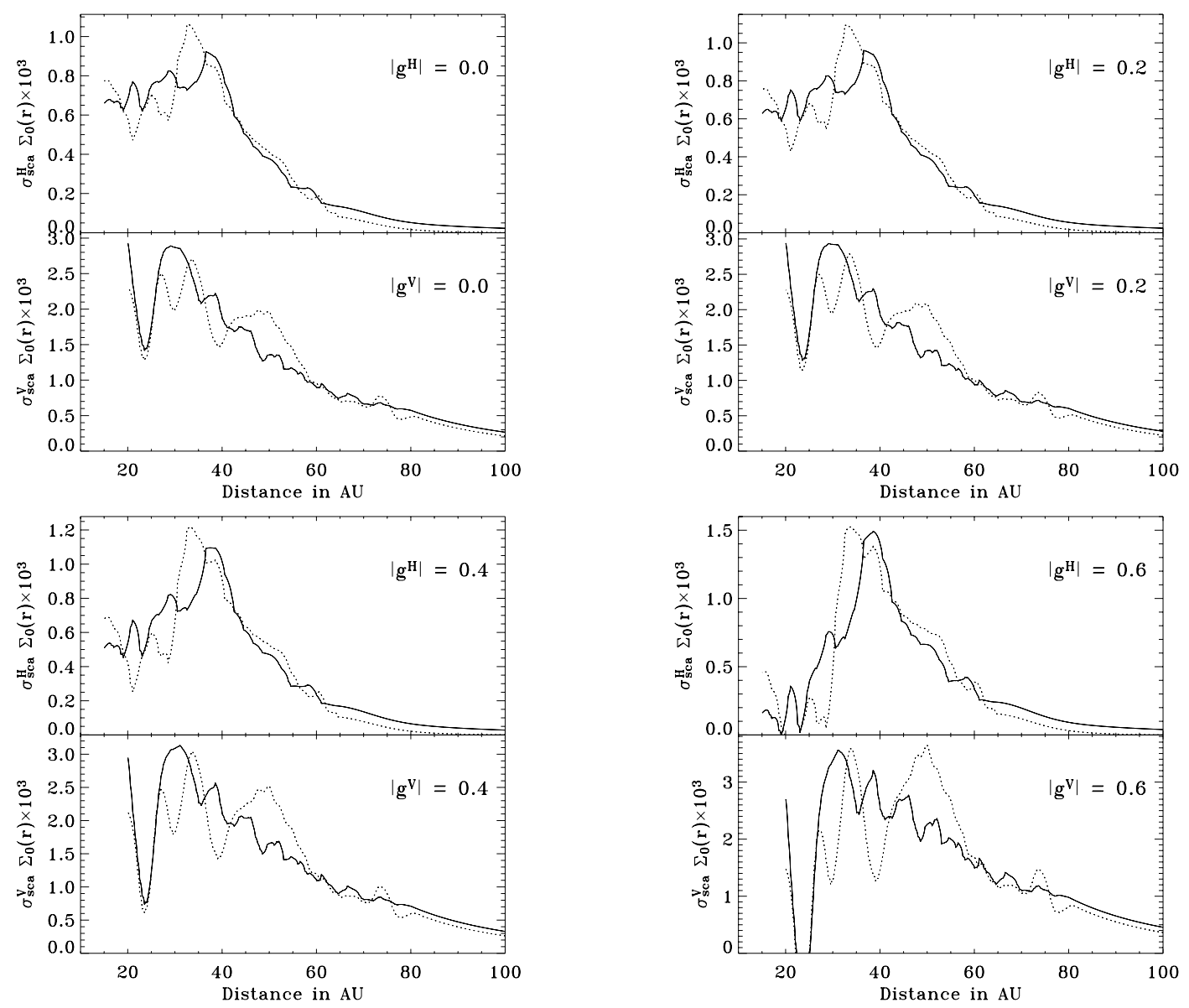

Fig. 3. Surface density profiles obtained by inverting the Liu (2004) $H$-band observations and the Krist et al. (2005) HST/ACS visible observations of the AU Mic disk (top and bottom panels, respectively, for each figure), assuming four mean scattering asymmetry parameters. The solid line corresponds to the NW side; the dotted line to the SE side. These results have been obtained assuming $\delta=1$ (see Sect. 3.3 and Appendix B), but the profiles are poorly sensitive to the assumed azimuthal profile provided $\delta \lesssim 5$.

They basically reflect the differences between the original images (see discussion in Krist et al. 2005). Along a given arm, the same density features can generally be identified (though often shifted by 1-2 AU with respect to each other), but not with the same strength. For instance, the NW surface density profiles inferred from the $H$-band observations peak at $\sim 37 \mathrm{AU}$, and show a smaller peak around $30 \mathrm{AU}$, while the feature strengths are reverted in the case of the surface density profiles obtained from the visible images. These two peaks correspond to features (D) and (C), respectively, according to the nomenclature of Liu (2004), and feature (D) is only marginally resolved in the ACS images. Along the SE side, features (A) and (B) around 25-27 AU and 33-34 AU, respectively, show strength ratios that depend on $|g|$ and on the data set used to calculate the disk surface density. In addition, the visible images harbor an extended clump around $50 \mathrm{AU}$ that results in a broad feature at that distance, and which is only marginally present in the surface density profiles deduced from the $H$-band observations. Almost all profiles, nevertheless, show a common dust depletion around 22-24 AU, the exact position depending on the data set used to calculate the surface density profiles. Besides the differences, we note that all profiles show complex structures that can hardly be approximated with simple power laws.

The differences between the surface density profiles derived from the visible and infrared observations may result from a combination of instrumental effects and grains optical properties varying throughout the disk midplane. The inversion of additional observed profiles and more constraints on the grain properties would help to disentangle these two possibilities. But if we assume that the differences are real, then the ratio $\sigma_{\mathrm{sca}}^{V} \Sigma_{0}(r) / \sigma_{\mathrm{sca}}^{H} \Sigma_{0}(r)$ is an estimate of the $V$ - to $H$-band scattering cross section ratio as a function of the distance. This ratio is plotted in Fig. 4 for the case $\left|g^{H}\right|=\left|g^{V}\right|=0.4$. This suggests that the $\sigma_{\text {sca }}^{V} / \sigma_{\text {sca }}^{H}$ ratio gradually increases beyond $\sim 40 \mathrm{AU}$, in line with the color gradient of the disk over the same region (Krist et al. 2005). Qualitatively, this would indicate that the large grains become less and less abundant, relative to the smallest grains at the same position, with increasing distance from the star. This behavior is expected if the grains have not been produced locally, but placed in orbits of high eccentricity by a pressure force $\propto s^{2} /\left(r^{2}+z^{2}\right)$ (where $s$ is the grain size), as shown in Fig. 4 of Augereau et al. (2001). We discuss the origin of pressure forces in the case of AU Mic in Sects. 5 and 6.

\section{Grain and disk properties}

\subsection{Disk color and grain size distribution}

The output of the inversion procedure is not the actual surface density profile of the disk, $\Sigma_{0}(r)$, but its product by the mean scattering cross section $\sigma_{\text {sca }}$. To evaluate $\sigma_{\text {sca }}$, and hence $\Sigma_{0}(r)$, we search for the grain properties that can reproduce the blue color of the AU Mic disk reported by Krist et al. (2005) in the HST/ACS bands. These authors find $F 435 W / F 606 W$ flux 
Table 1. Grain properties inferred from the interpretation of the disk colors in the visible (Krist et al. 2005) and implications for the disk mass.

\begin{tabular}{|c|c|c|c|c|c|c|}
\hline \multirow[b]{2}{*}{ Description } & \multirow[b]{2}{*}{ Parameter $^{a}$} & \multicolumn{2}{|c|}{ ASTRONOMICAL SILICATES } & \multicolumn{2}{|c|}{ GRAPHITE GRAINS } & \multirow[b]{2}{*}{ [Unit] } \\
\hline & & NW & SE & NW & SE & \\
\hline Nominal minimum grain size & $s_{\min }$ & 0.115 & 0.123 & 0.0583 & 0.0609 & \multirow[t]{4}{*}[\mu\mathrm{m}]{} \\
\hline Nominal power law index & $\kappa$ & -3.74 & -4.01 & -3.95 & -4.18 & \\
\hline$H$-band mean asymmetry factor & $\left|g^{H}\right|$ & 0.637 & 0.604 & 0.358 & 0.318 & \\
\hline$V$-band mean asymmetry factor & $\left|g^{V}\right|$ & 0.675 & 0.661 & 0.455 & 0.421 & \\
\hline$H$-band mean scattering cross section & $\sigma_{\mathrm{sca}}^{H}$ & 16.0 & 12.7 & 2.12 & 1.64 & \multirow[t]{3}{*}[10^{-10}\mathrm{cm}^{2}]{} \\
\hline Ratio of mean cross sections & $\sigma^{V} / \sigma^{H}$ & 2.05 & 2.56 & 2.15 & 2.65 & \\
\hline$V$-band mean albedo & $\omega^{V}$ & 0.795 & 0.822 & 0.518 & 0.513 & \\
\hline \multirow{4}{*}{$\begin{array}{l}\text { Disk scattering cross section } \\
\text { Dust mass }^{c} \\
\text { TOTAL DUST DISK MASS }{ }^{c}\end{array}$} & & \multicolumn{4}{|c|}{$H$-BAND DATA SET } & \multirow{4}{*}{$\begin{array}{l}{\left[\mathrm{AU}^{2}\right]} \\
{\left[10^{-4} M_{\oplus}\right]} \\
{\left[10^{-4} M_{\oplus}\right]}\end{array}$} \\
\hline & \multirow[t]{2}{*}{$S_{\mathrm{sca}}^{H}$} & $4.93-6.97$ & $4.51-6.30$ & $4.93-6.97$ & $4.51-6.30$ & \\
\hline & & $2.60-3.67$ & $0.952-1.33$ & $0.583-0.824$ & $0.306-0.428$ & \\
\hline & $M_{\text {disk }}$ & \multicolumn{2}{|c|}{$3.55-5.00$} & \multicolumn{2}{|c|}{$0.889-1.25$} & \\
\hline \multirow{4}{*}{$\begin{array}{l}\text { Disk scattering cross section } \\
\text { Dust mass }^{c} \\
\text { TOTAL DUST DISK MASs }^{c}\end{array}$} & \multirow{3}{*}{$S_{\mathrm{sca}}^{V}$} & & $V$-BA & LTA SET & & \multirow{4}{*}{$\begin{array}{l}{\left[\mathrm{AU}^{2}\right]} \\
{\left[10^{-4} M_{\oplus}\right]} \\
{\left[10^{-4} M_{\oplus}\right]}\end{array}$} \\
\hline & & $15.9-23.7$ & $15.5-23.1$ & $15.9-23.7$ & $15.5-23.1$ & \\
\hline & & $4.10-6.10$ & $1.28-1.91$ & $0.876-1.31$ & $0.398-0.593$ & \\
\hline & $M_{\text {disk }}$ & \multicolumn{2}{|c|}{$5.38-8.01$} & \multicolumn{2}{|c|}{$1.27-1.90$} & \\
\hline
\end{tabular}

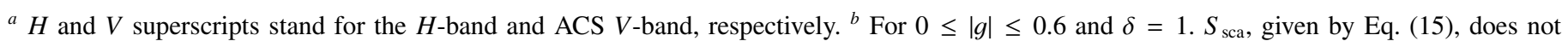
depend on the assumed grain composition. ${ }^{c}$ Mass inferred from the analysis of the scattered light observations. See Sect. 4.3 for an estimate of the dust mass deduced from the analysis of the disk thermal emission. Mass obtained assuming $s_{\max }=1 \mathrm{~mm}, \rho_{\text {Silicates }}=3.5 \mathrm{~g} \mathrm{~cm}{ }^{-3}$, and $\rho_{\text {graphite }}=2.2 \mathrm{~g} \mathrm{~cm}^{-3}$. The total dust disk mass is the sum of the NW and SE dust masses.

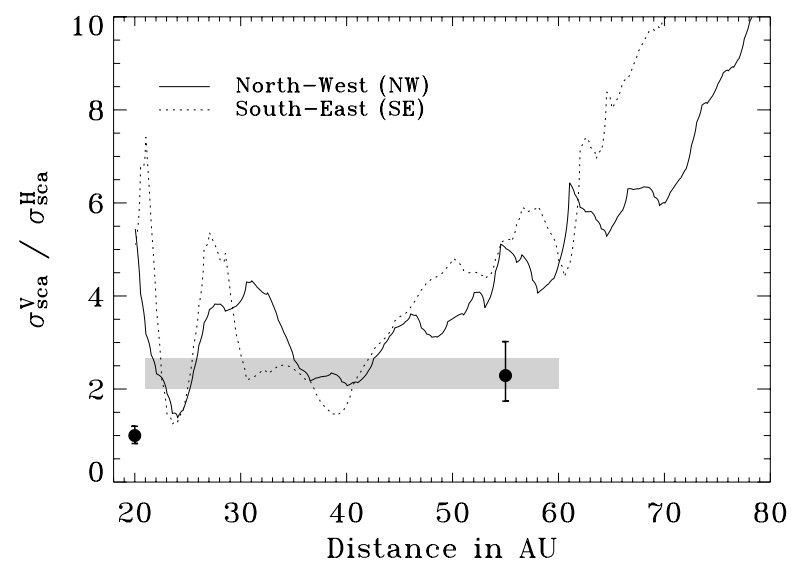

Fig. 4. Scattering cross section ratio $\sigma_{\text {sca }}^{V} / \sigma_{\text {sca }}^{H}$ obtained by dividing the profiles calculated for $\left|g^{H}\right|=\left|g^{V}\right|=0.4$ and shown in Fig. 3. The gray area corresponds to the range of scattering cross section ratios inferred from the analysis of the disk color in the ACS bands (Sect. 4.1 and Table 1). The $R-H$ colors inferred by Metchev et al. (2005) are displayed as two black dots at 20 and $55 \mathrm{AU}$.

ratios, relative to the star and averaged over a $43 \mathrm{AU}$ long aperture centered at $r=42 \mathrm{AU}$, of $1.13 \pm 3 \%$ and $1.15 \pm 3 \%$ for the NW and the SE sides of the disk, respectively. The measured $F 435 W / F 814 W$ flux ratios amount to $1.35 \pm 3 \%$ (NW) and $1.44 \pm 3 \%$ (SE). Qualitatively, the blue color indicates that the visible scattered light images are dominated by elementary (sub-)grains of the order of or smaller than a few tenths of micrometers.

Provided the phase functions are not too different, the measured colors are only dependent upon the ratios of mean scattering cross sections in the ACS passbands. We considered optical constants for astronomical silicates (Weingartner \& Draine 2001) and graphite grains (Laor \& Draine 1993), and we used the Mie theory, appropriate for hard spheres, to calculate the scattering efficiency $Q_{\text {sca. }}$. We assumed a differential grain size distribution of the form $\mathrm{d} n(s) \propto s^{\kappa} \mathrm{d} s$ with $\kappa<0$ between $s_{\min }$ and $s_{\max }$. The maximum grain size is assumed to be large enough not to affect the results. The scattering cross sections were calculated using Eq. (10).

We have searched for the pairs $\left(s_{\min }, \kappa\right)$ that give scattering cross section ratios equal to the two HST/ACS colors. Each side of the disk has been considered independently. The results are reported in Table 1 and in Fig. 5, where the cross sign indicates the nominal couple $\left(s_{\min }, \kappa\right)$, namely the couple that produces a perfect match to the two ACS disk colors. For astronomical silicates, the blue color of the disk is reproduced when the minimum grain size $s_{\min }$ is smaller than $0.14 \mu \mathrm{m}$, with a nominal $s_{\min }$ value of $0.12 \mu \mathrm{m}$. For graphite grains, we find $s_{\min }<0.07 \mu \mathrm{m}$, with a nominal $s_{\min } \simeq 0.06 \mu \mathrm{m}$. For a given grain composition, our simple model predicts quite similar nominal $s_{\min }$ values for both the SE and NW sides of the disk, but the nominal slopes $\kappa$ differ: the size distribution in the NW side is found to be less steep than the SE size distribution where the disk is found to be even bluer (Krist et al. 2005). $\kappa$ values consistent with the HST/ACS colors are generally found to be slightly smaller than the canonical -3.5 value that holds for systems in collisional equilibrium (Dohnanyi 1969). The minimum grain sizes we infer for astronomical silicates are smaller than the $s_{\min }$ value of $0.5_{-0.2}^{+0.5} \mu \mathrm{m}$ obtained by Metchev et al. (2005) based on the $R-H$ color of the disk measured around 50-60 AU and assuming $\kappa=-3.5$.

The mean asymmetry factors reported in Table 1 were calculated using Eq. (9). For astronomical silicates, $|g|$ reaches or slightly overcomes the 0.6 limit derived from the inversion of the scattered light profiles (Sect. 3.4). For more refractory grains, such as graphite grains, the mean asymmetry factor remains smaller than the $|g|=0.6$ limit and is close, in the $V$-band, to the 0.4 value inferred by Krist et al. (2005) from the disk modeling.

\subsection{Disk optical thickness}

In the previous sections, we calculated the surface density and grain properties of the AUMic disk, assuming an optically thin medium at all wavelengths and in all directions. This assumption has been justified in Sect. 2.1 by the low fractional disk luminosity and the appearance of the disk at visible and 

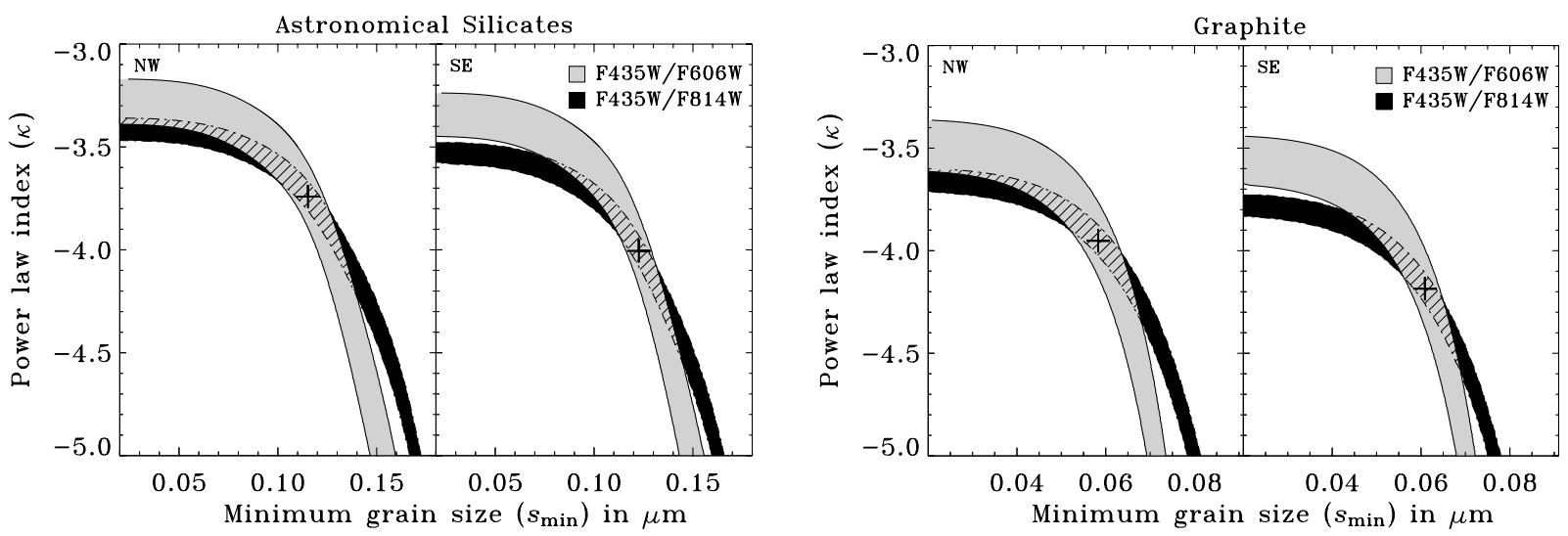

Fig. 5. Minimum grain sizes $s_{\min }$ and power-law indexes $\kappa$ of the size distribution consistent with the HST/ACS colors measured by Krist et al. (2005). Gray area: $F 435 W / F 606 W$, dark area: $F 435 W / F 814 W$, dashed area: intersection. The cross sign corresponds to the nominal pair $\left(s_{\text {min }}, \kappa\right)$ that produces a perfect match to the two colors. The nominal values are given in Table 1.

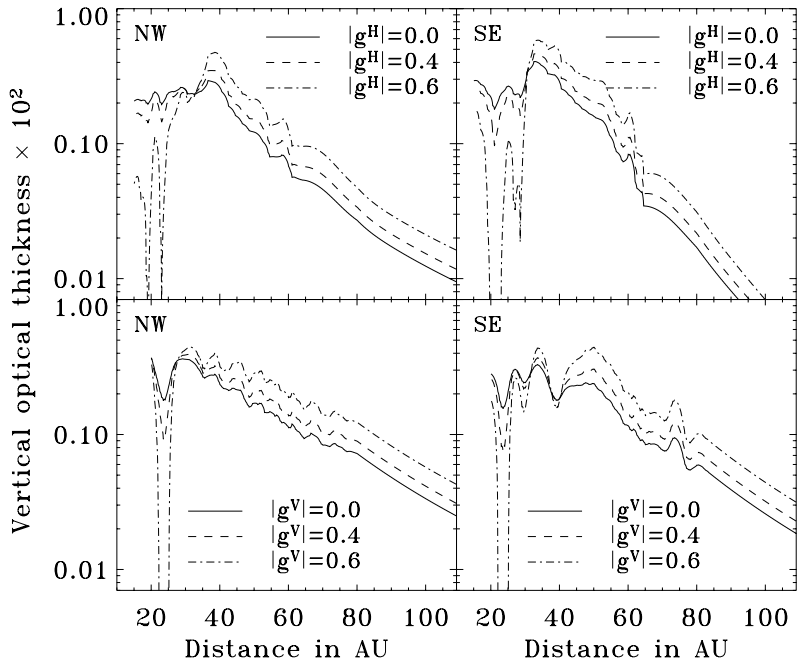

Fig. 6. Vertical optical thickness assuming astronomical silicates (see Table 1). Top panels: surface density profiles inferred from the inversion of the $H$-band observations; bottom panels: surface density profiles derived from $V$-band observations.

near-IR wavelengths. We verify in the following that our results are self-consistent. reads

The vertical optical thickness of the AUMic disk, $\tau_{\perp}(r)$,

$\tau_{\perp}(r)=\frac{1}{\omega^{V}} \times \sigma_{\mathrm{sca}}^{V} \Sigma_{0}(r)=\frac{\sigma_{\mathrm{sca}}^{V}}{\omega^{V} \sigma_{\mathrm{sca}}^{H}} \times \sigma_{\mathrm{sca}}^{H} \Sigma_{0}(r)$,

where $\omega^{V}$ is the albedo of the dust particles in the $V$-band averaged over the grain size distribution. The ratio $\sigma_{\text {sca }}^{V} / \sigma_{\text {sca }}^{H}$ and the mean albedo $\omega^{V}$ have been estimated assuming the grain properties summarized in Table 1 . In the visible, the disk vertical thickness $\tau_{\perp}(r)$ does not exceed $\sim 5 \times 10^{-3}$, indicating that the disk is optically thin in the vertical direction (Fig. 6). We checked that with the assumed grain properties, the vertical thickness is maximum in the visible, implying $\tau_{\perp} \ll 1$ at all wavelengths.

The midplane density of the disk is written $\Sigma_{0}(r) /(\pi H(r))$, and the optical thickness along the disk midplane thus reads

$\tau_{\|}(r)=\int_{r_{\min }}^{r} \frac{\sigma_{\text {sca }}^{V}}{\omega^{V}} \frac{\Sigma_{0}(r)}{\pi H(r)} \mathrm{d} r=\int_{r_{\min }}^{r} \frac{\tau_{\perp}(r)}{\pi H(r)} \mathrm{d} r$,

where $r_{\text {min }}=15 \mathrm{AU}$ in the $H$-band and $r_{\text {min }}=20 \mathrm{AU}$ in the ACS $V$-band (see Figs. 2 and 3). Figure 7 shows that $\tau_{\|}(r)$ does not

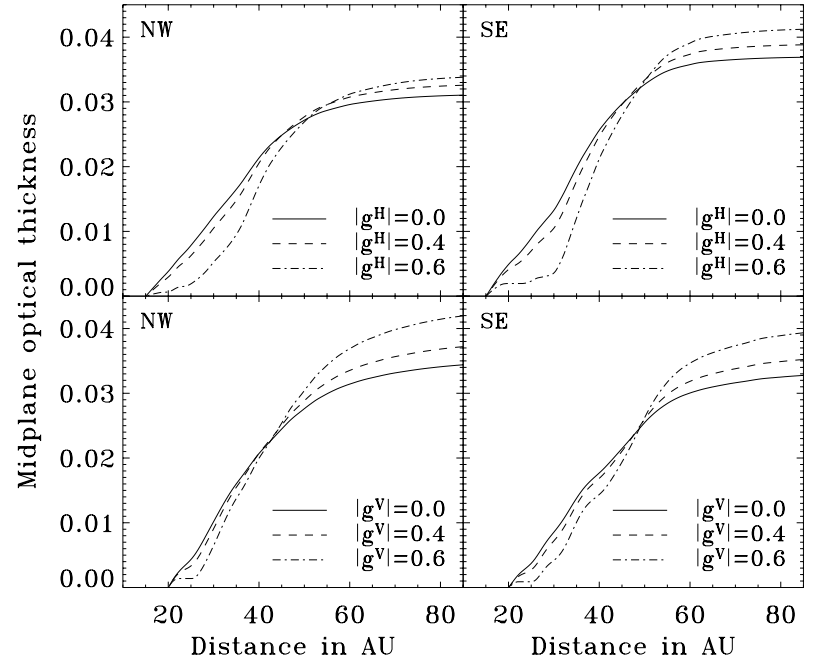

Fig. 7. Midplane optical thickness assuming the same grain properties as in Fig. 6.

exceed $\sim 4 \times 10^{-2}$ in the visible, whenever $|g| \leq 0.6$, and this result is independent of the data set used to calculate the surface density profiles. Given that $\tau_{\perp}$, and thus $\tau_{\|}$, reach their maxima at visible wavelengths in this model, these results indicate that the disk is optically thin in all directions and at all wavelengths.

\subsection{Disk mass and implication for the grain properties}

From the surface density profiles calculated in Sect. 3.4 and the constraints on the grain size distribution obtained in Sect. 4.1, we can estimate the mass of the dust disk $M_{\text {disk }}$ further out than $r_{\min }$ :

$M_{\mathrm{disk}}=\frac{S_{\mathrm{sca}}(|g|)}{\sigma_{\mathrm{sca}}} \times \int_{s_{\min }}^{s_{\max }} \rho \frac{4 \pi}{3} s^{3} \mathrm{~d} n(s)$,

where $\rho$ is the grain mass density. The disk scattering cross section $S_{\text {sca }}(|g|)$ is

$S_{\mathrm{sca}}(|g|)=\int_{r=r_{\min }}^{r_{\max }} \int_{\theta=0}^{2 \pi} \sigma_{\mathrm{sca}} \Sigma(r, \theta) r \mathrm{~d} r \mathrm{~d} \theta$,

where $r_{\max }$ was fixed to $140 \mathrm{AU}$. The results are given in Table 1.

The total mass of dust derived from the scattered light analysis is of the order of the mass of the Ceres asteroid $(\sim 1.46 \times$ $\left.10^{-4} M_{\oplus}\right)$ in the Solar System, to within a few times. This is 
about 10 to 80 times the estimated dust mass in the Kuiper Belt $\left(\sim 10^{-5} M_{\oplus}\right.$, Backman et al. 1995). But it is significantly smaller than the dust mass calculated by Liu et al. (2004) from submillimeter observations $\left(1.1 \times 10^{-2} M_{\oplus}\right)$. In fact, a dust mass as low as a few $10^{-4} M_{\oplus}$ is not expected at an age of about $10 \mathrm{Myr}$ (e.g. Najita \& Williams 2005).

To help identify the origin of the mass difference, we calculated the disk Spectral Energy Distribution (SED), assuming the surface density profiles and the grain properties obtained from the analysis of the scattered light observations. We assumed the star is in a quiescent period (no UV-flares, see Fig. 9 and Sect. 5.2). We find that the total $25 \mu \mathrm{m}$ flux is always well reproduced, while the $60-70 \mu \mathrm{m}$ fluxes are systematically underestimated by factors of 1.5 to 2 . More critically, the observed sub-millimeter emissions are 10 to 20 times larger than predicted when silicates are considered. These flux ratios amount to 15 to 30 for the graphite grains.

On the other hand, we find that the whole disk SED can be reproduced provided the constraints on the grain size distribution are relaxed. Good fits to the SED are obtained for $s_{\min } \simeq 1.0 \mu \mathrm{m}$ and $\kappa \simeq-3.4$, when silicate grains and the surface density profiles derived from the $H$-band images are assumed (Fig. 9). With the $V$-band surface density profiles, we find $s_{\min } \simeq 0.1 \mu \mathrm{m}$ and $\kappa \simeq-3.4$. In both cases, the dust disk mass is approximately $7 \times 10^{-3} M_{\oplus}$, in better agreement with the submillimeter dust mass $\left(1.1 \times 10^{-2} M_{\oplus}\right)$ calculated by Liu et al. (2004), assuming a single temperature formula.

The grain size distributions that reproduce the disk colors in scattered light do, therefore, underestimate the number of large grains in the disk, which explains the disagreement between the two independent mass estimates. It might be that the dust properties in Table 1 are only valid in a small range of grain sizes. This could be the case, for instance, if the actual grain size distribution departs from a simple power law, as is expected for systems in collisional equilibrium (see discussion in Thébault et al. 2003). It might also be possible that the grains have complex structures, and hence optical properties, that cannot be consistently reproduced over the whole spectrum, assuming hard spheres.

\section{Diffusion of dust particles in the outer disk. I. Time-variable radiation forces}

Radiation pressure is a key parameter controlling the dynamics of dust particles in usual debris disks. The grains are thought to be produced by numerous colliding or evaporating planetesimals orbiting the star on nearly circular orbits. Depending on their size, the radiation pressure can transport the grains far away from their production zone and fill the outer regions of the disk. This model explains the observed radial profiles of the $\beta$ Pictoris and HD 181327 disks, for instance (Lecavelier des Étangs et al. 1996; Augereau et al. 2001; Thébault \& Augereau 2005; Schneider et al. 2006). We discuss below and in the next section whether this model could apply to the AU Mic disk.

\subsection{Equation of motion and dynamical time scales}

A dust particle of mass $m$ and geometric cross section $\sigma$ feels a full force from the stellar radiation field of energy flux $F$ that reads

$\left.m \frac{\mathrm{d}^{2} \boldsymbol{r}}{\mathrm{d} t^{2}}\right|_{\mathrm{PR}}=\frac{F \sigma Q_{\mathrm{pr}}}{c}\left[\left(1-\frac{\dot{r}}{c}\right) \hat{\boldsymbol{u}}-\frac{\boldsymbol{v}}{c}\right]$
(Burns et al. 1979), where $\boldsymbol{r}$ is the position vector of the particle with respect to the star, $\boldsymbol{v}$ its velocity vector, $\hat{\boldsymbol{u}}=\boldsymbol{r} / \boldsymbol{r}$ is the radial unit vector, $\dot{r}=\hat{\boldsymbol{u}} \cdot \boldsymbol{v}$ is the radial velocity, and $c$ is the speed of light; $Q_{\mathrm{pr}}$ is the dimensionless radiation pressure efficiency coefficient averaged over the stellar spectrum. In this expression, the velocity independent term $\left(F \sigma Q_{\mathrm{pr}} / c\right) \hat{\boldsymbol{u}}$ is often referred to as the "radiation pressure", while the other terms constitute the Poynting-Robertson drag (hereafter PRD) (Gustafson 1994). The radiation pressure is radial, and it is usually described by its ratio $\beta_{\mathrm{pr}}$ to stellar gravity $F_{\mathrm{g}}=G M m / r^{2}$, where $M$ is the mass of the star and $G$ the gravitational constant. Since $F \propto r^{-2}$, $\beta_{\mathrm{pr}}$ does not depend on $r$ for a given particle. Equation (16) is then rewritten as

$\left.\frac{\mathrm{d}^{2} \boldsymbol{r}}{\mathrm{d} t^{2}}\right|_{\mathrm{PR}}=\frac{\beta_{\mathrm{pr}} G M}{r^{2}}\left[\left(1-\frac{\dot{r}}{c}\right) \hat{\boldsymbol{u}}-\frac{\boldsymbol{v}}{c}\right]$.

Since $v \ll c$, the radiation pressure largely overcomes the PRD.

The long-term effect of PRD is to cause a decrease of the orbit. Computing the rate of change of the specific angular momentum due to PRD, and assuming that the orbit is circular, we derive the orbital decrease as

$\frac{\mathrm{d} r}{\mathrm{~d} t}=-\frac{2 \beta_{\mathrm{pr}} G M}{r c} \equiv-2 \frac{\alpha}{r}$,

where $\alpha$ is a constant parameter. Numerically, we have

$\alpha=\beta_{\mathrm{pr}} \times 6.24 \times 10^{-4} \frac{M}{M_{\odot}} \mathrm{AU}^{2} \mathrm{yr}^{-1}$,

a value already quoted by Wyatt (2005). The characteristic PRD time scale is written

$t_{\mathrm{PRD}}=|r /(\mathrm{d} r / \mathrm{d} t)|=0.5 r^{2} / \alpha$.

As we see below, $\beta_{\mathrm{pr}}$ barely reaches 0.2 for submicron-sized particles. Assuming $\beta_{\mathrm{pr}}=0.1$ and $M=0.59 M_{\odot}$ (Houdebine $\&$ Doyle 1994), the PRD time scale turns out to range between $2.7 \times 10^{6} \mathrm{yr}$ at $r=20 \mathrm{AU}$ and $7 \times 10^{7} \mathrm{yr}$ at $100 \mathrm{AU}$. As quoted by Kalas et al. (2004), at sufficiently large distances, it is longer than the age of the system $\left(12_{-4}^{+8} \mathrm{Myr}\right.$, Barrado y Navascués et al. 1999; Zuckerman et al. 2001).

The PRD time scale, moreover, needs to be compared to the collisional time scale, as collisions between grains of various sizes tend to prevent the orbital decrease of those for which PRD is more efficient. The collision time scale may be estimated as

$t_{\mathrm{coll}} \simeq \frac{\sigma_{\mathrm{sca}}}{\pi\left\langle s^{2}\right\rangle} \times \frac{r^{3 / 2}}{2 \sigma_{\mathrm{sca}} \Sigma_{0}(r) \sqrt{G M}}$,

where $\pi\left\langle s^{2}\right\rangle$ is the mean geometric cross-section of the grains averaged over the grain size distribution (Backman \& Paresce 1993). The result is shown in Fig. 8 for astronomical silicates. We see that for any distance $r$, the collision time scale is more than one order of magnitude less that the PRD time scale. Hence, we may safely stress that collisions dominate over PRD; if any effect of PRD is to be investigated, it must be averaged over the size distribution, assuming an average $\beta_{\mathrm{pr}} \simeq 0.01$. The corresponding PRD time scale turns out to be larger than the age of the star, so that we may assume that PRD is of negligible importance in the dynamics of the AU Mic disk. Actually, the same conclusion is derived by Wyatt (2005) for all the debris disks known today.

Assuming the Poynting-Robertson drag force on the grains is negligible in the AU Mic disk, the equation of motion of a grain thus reads

$\frac{\mathrm{d}^{2} \boldsymbol{r}}{\mathrm{d} t^{2}}=-\frac{G M\left(1-\beta_{\mathrm{pr}}\right)}{r^{2}} \hat{\boldsymbol{u}}$. 


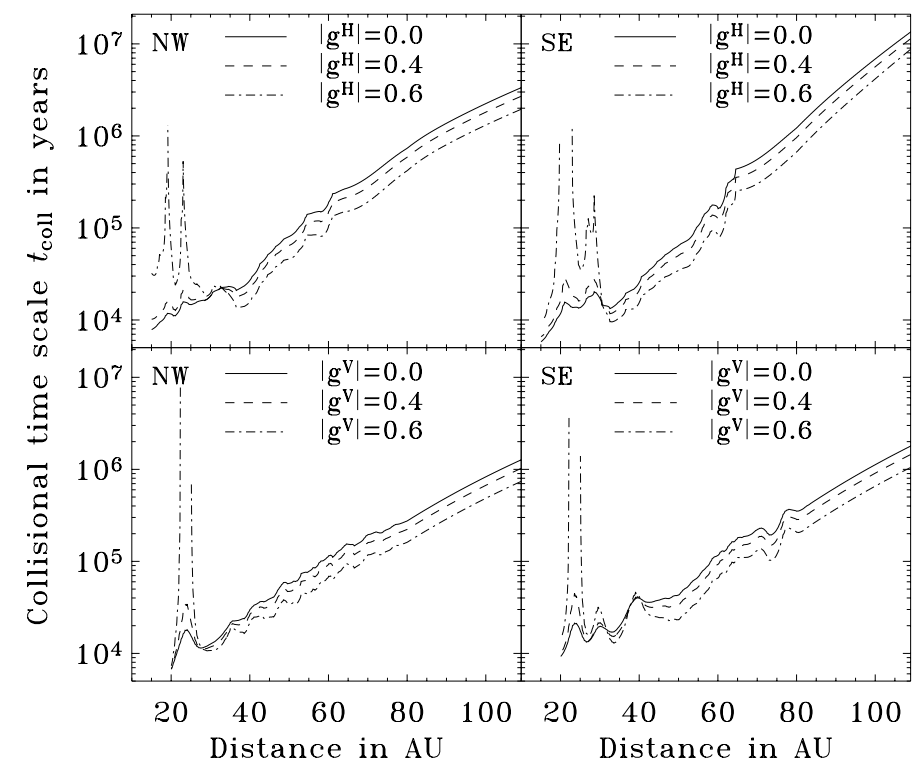

Fig. 8. Collisional time scale assuming the surface densities displayed in Fig. 3 and the grain size distribution for astronomical silicates from Table 1 .

This is a classical equation of a Keplerian motion. While suffering a radiation pressure force, a particle keeps following a Keplerian orbit around the star, but with an effective central mass $M\left(1-\beta_{\mathrm{pr}}\right)$. Depending on the value of $\beta_{\mathrm{pr}}$, that orbit may be very different from the original orbit of the parent body. It is eccentric and shares the same periastron as the parent body orbit, but has a very different apoastron. At release time, the grain assumes the same orbital velocity as its parent body. If the orbit of the parent body is circular, energy and angular momentum preservation cause the grain orbit to obey

$a^{\prime}=\frac{a\left(1-\beta_{\mathrm{pr}}\right)}{1-2 \beta_{\mathrm{pr}}}, \quad e^{\prime}=\frac{\beta_{\mathrm{pr}}}{1-\beta_{\mathrm{pr}}}, \quad Q^{\prime}=\frac{a}{1-2 \beta_{\mathrm{pr}}}$.

Here, $a$ is the semi-major axis of the original orbit, and $a^{\prime}$ is that of the resulting dust particle orbit; $e^{\prime}$ is the eccentricity of that orbit and $Q^{\prime}$ is its apoastron. If $\beta_{\mathrm{pr}}$ is sufficiently close to 0.5 , the grain is transported at a large distance, thanks to the large $Q^{\prime}$ value. If $\beta_{\mathrm{pr}}>0.5$, it is expelled from the system.

\subsection{The spectrum of a AUMic, a flare star}

The $\beta_{\mathrm{pr}}$ ratio depends on the grain properties (size, composition) and on the SED of the central star. For any given star, $\beta_{\mathrm{pr}}$ is maximum for grains with radius $s$ in the range $0.1-1 \mu \mathrm{m}$, falls off like $s^{-1}$ (geometric regime, $Q_{\mathrm{pr}}=$ const.) at larger sizes, and tends to reach a constant for smaller grain sizes $\left(Q_{\mathrm{pr}} \propto s\right.$, Rayleigh scattering regime, Gustafson 1994). In the solar case, $\beta_{\mathrm{pr}}$ barely reaches 1 for submicron-sized particles (Gustafson 1994; Beust et al. 2001), but in the case of classical Vega-like stars (A-type stars), $\beta_{\text {pr }}$ reaches high enough values to enable ejection (a few micrometers in the case of $\beta$ Pictoris, depending somehow on the grain composition, e.g., Artymowicz 1988; Beust et al. 2001; Augereau et al. 2001).

Contrary to those stars, AU Mic is an M-type star; hence, radiation pressure is expected to be weak (Kalas et al. 2004; Liu 2004; Krist et al. 2005). To compute it, we need to model the SED of the star. We fit the stellar spectrum with a NEXTGEN model (Hauschildt et al. 1999) with $\log (g)=4.5$ (CGS) and an effective temperature of $3700 \mathrm{~K}$. This gives a stellar luminosity of $0.092 L_{\odot}$. However, this model is not enough to describe the full SED of AU Mic.

AUMic is known to frequently present X-ray and EUV flares (Kunkel 1973; Cully et al. 1993; Tsikoudi \& Kellett 2000; Magee et al. 2003). The flaring rate is estimated by Kunkel (1973) at 0.9 per hour. During these flares, the EUV and X-ray luminosities increase by a typical factor 10 (Cully et al. 1993; Monsignori-Fossi et al. 1996; Tsikoudi \& Kellett 2000). We may estimate that the star spends $\sim 10 \%$ of the time in the flaring state. Even in a quiescent state, the coronal activity of the star is not negligible. Tsikoudi \& Kellett (2000) note $L_{X}=$ $2.24 \times 10^{29} \mathrm{erg} \mathrm{s}^{-1}$ and $L_{\mathrm{EUV}}=2.9 \times 10^{28} \mathrm{erg} \mathrm{s}^{-1}$ for the $\mathrm{X}$-ray and EUV luminosities, respectively. Hünsch et al. (1999) give $L_{X}=$ $5.5 \times 10^{29} \mathrm{erg} \mathrm{s}^{-1}$. Mitra-Kraev et al. (2005) report simultaneous X-ray and UV observations of AU Mic with XMM-Newton and give luminosities in both domains. Their X-ray luminosity is compatible with the previous estimate of Tsikoudi \& Kellett (2000). These values are far above what should be expected from the stellar model described above. The X-ray and EUV luminosities may contribute to the radiation pressure felt by the dust particles, so that it is necessary to take them into account in the stellar SED model we use. The global shape of the $\mathrm{X}$-ray and EUV spectra of AU Mic is given in Monsignori-Fossi \& Landini (1994); in the UV spectral domain between $1150 \AA$ and $2000 \AA$ A IUE data are available (Landsman \& Simon 1993); Archive HST/FOS spectra cover the spectral domain between $1600 \AA$ and $6800 \AA$. Finally, broadband data $(U, B, V, R \mathrm{c}, I \mathrm{c}$, 2MASS, IRAS, Spitzer, SHARC II, and SCUBA) provide additional measurements towards the infrared (Chen et al. 2005, and references therein).

Given these constraints, we fit the full spectrum of AU Mic by adding the flux,

$$
F(\lambda)=\exp \left[a_{0}+\frac{a_{5}}{1+\left(\frac{\ln (\lambda)-a_{1}}{a_{2}}\right)^{2}}+\frac{a_{3}}{\lambda}-\frac{a_{3} a_{4}}{2 \lambda^{2}}\right](\lambda \text { in } \AA)
$$

in Jansky at Earth, to the NEXTGEN stellar atmosphere model with

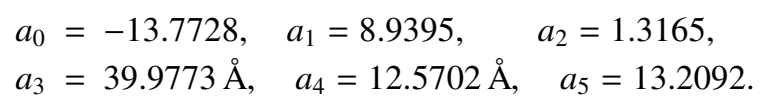

This function accurately fits the various data in the UV and EUV domains and is compatible with the X-ray and EUV luminosities given by Tsikoudi \& Kellett (2000). This holds for the quiescent state. During flares, we expect the flux in X-ray and UV to be higher. There is, nevertheless, good evidence for synchronicity between flare events in X-ray and in UV (Tsikoudi \& Kellett 2000; Mitra-Kraev et al. 2005), the time lag between the two spectral domains being less than a few minutes (Mitra-Kraev et al. 2005). Hence, we decided to mimic flare spectra by multiplying the $F(\lambda)$ function we add to the NEXTGEN model by a fixed, average factor we estimate to be 10 (see Tsikoudi \& Kellett 2000), for wavelengths shorter than $2500 \AA^{1}$. The result is displayed in Fig. 9, where we superimpose the fits on the NEXTGEN model and on the various measurements. We see that shortwards of $\sim 0.3 \mu \mathrm{m}$, the resulting spectrum is significantly above the NEXTGEN model and will have an influence on the $\beta_{\mathrm{pr}}$ parameter that is felt by the various grains.

1 In practice, we multiplied Eq. (23) by $10 \times(0.5-\arctan ((\lambda-$ 2500)/500)/ $\pi$ ) with $\lambda$ in $\AA$. 


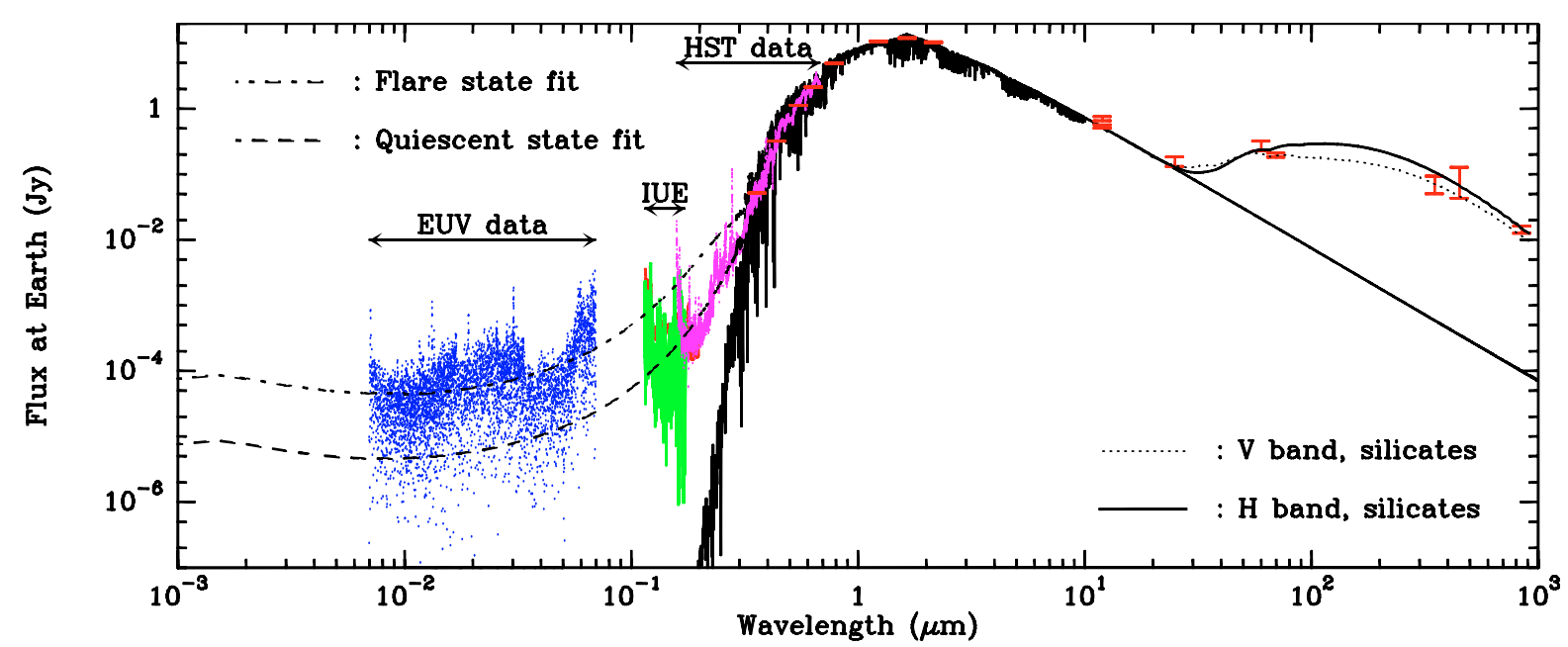

Fig. 9. Observed SED of the AU Mic system. The solid black curve corresponds to the NEXTGEN stellar atmosphere spectrum that fits the visible and near-infrared data. The dashed and dashed-dotted curves are the SED fits for the quiescent and flare states respectively (Eq. (23)). Two illustrative fits to the disk SED are also overplotted, assuming the two surface density profiles obtained for $|g|=0.4$ (see Sect. 4.3 ) and silicate grains.

\subsection{Radiation pressure during $X$-ray and UV flares}

As the X-ray and UV spectrum of AU Mic is subject to fluctuations due to flares, the $\beta_{\mathrm{pr}}$ ratio for a given grain may be timevariable. Hence, the dynamics of the dust particles may be more complex than described above, where we assumed a constant $\beta_{\mathrm{pr}}$ value. As the flares are very frequent, we cannot neglect this effect. Thus, we now investigate the dynamics of solid particles with a time-variable $\beta_{\mathrm{pr}}$.

In a simple description, we assume that the star periodically alternates quiescent and flare phases, respectively characterized for a given grain by $\beta_{\mathrm{pr}, \mathrm{q}}$ and $\beta_{\mathrm{pr}, \mathrm{f}}$ ratios, with $\beta_{\mathrm{pr}, \mathrm{f}}>\beta_{\mathrm{pr}, \mathrm{q}}$. The star is assumed to spend a fixed fraction $\gamma$ of the time in flare phases. Strictly speaking, the dust particle will follow bits of Keplerian orbits alternatively characterized by dynamical masses $M\left(1-\beta_{\mathrm{pr}, \mathrm{q}}\right)$ and $M\left(1-\beta_{\mathrm{pr}, \mathrm{f}}\right)$. It can be shown (see Appendix C) that the global effect is the same as if $\beta_{\mathrm{pr}}$ were constant, equal to its temporal mean. The dust particle keeps following a Keplerian orbit around the star, but is characterized by a $\beta_{\text {preff }}$ ratio equal to the temporal average of $\beta_{\mathrm{pr}}$, namely $\beta_{\mathrm{pr}, \mathrm{eff}}=(1-\gamma) \beta_{\mathrm{pr}, \mathrm{q}}+\gamma \beta_{\mathrm{pr}, \mathrm{f}}$.

Our problem is reduced, therefore, to estimating the $\beta_{\mathrm{pr}, \text { eff }}$ ratio, i.e., the parameters $\beta_{\mathrm{pr}, \mathrm{q}}, \beta_{\mathrm{pr}, \mathrm{f}}$, and $\gamma$. Figure 10 shows the value of $\beta_{\mathrm{pr}}$ as a function of the grain radius, computed from the SED of Fig. 9, in both quiescent and flare phases, for two grain compositions and assuming hard spheres. We also plot the $\beta_{\mathrm{pr}, \text { eff }}$ average, assuming $\gamma=0.1$. Figure 10 shows that unless the grains are very refractory, they do not suffer a radiation pressure force large enough, to a priori produce an extended dust disk in quiescent phases. For silicate grains, $\beta_{\mathrm{pr}}$ barely reaches 0.1 for submicron-sized grains. The radiation pressure is then only able to at most transport the grains up to $25 \%$ of their original distance $\left(Q^{\prime}=1.25 a\right.$ for $\left.\beta_{\mathrm{pr}}=0.1\right)$. To reach a distance of $210 \mathrm{AU}$ (maximal disk radius, Kalas et al. 2004), a grain produced at $40 \mathrm{AU}$ needs $\beta_{\mathrm{pr}} \simeq 0.405$, a value never reached during quiescent phases, even for refractories. Conversely, during flares, the $\beta_{\mathrm{pr}}$ ratio of graphite grains can reach 0.5 , but the temporal mean $\beta_{\mathrm{pr} \text {,eff }}$ does not. With $\gamma=0.1$ (estimated from the light curves of Tsikoudi \& Kellett 2000; Magee et al. 2003; Mitra-Kraev et al. $2005), \beta_{\mathrm{pr} \text { eff }}$ remains close to $\beta_{\mathrm{pr}, \mathrm{q}}$, and the conclusion derived above during quiescent phases still holds. The flares help diffuse the particles slightly further out, but not enough to explain the

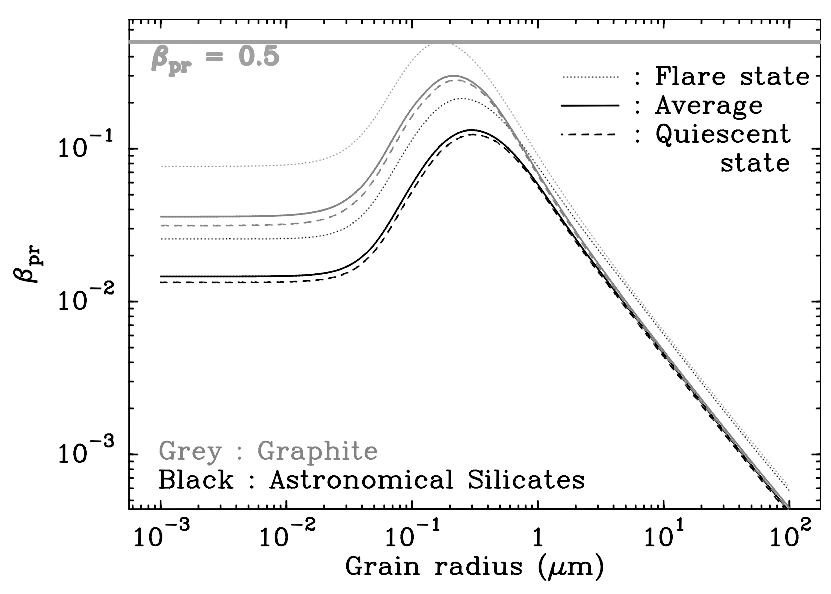

Fig. 10. $\beta_{\text {pr }}$ ratio for astronomical silicates (black curves) and graphite grains (grey curves) assumed to be spherical and homogeneous, as a function of their radius. The dashed curves correspond to the quiescent state of the star, the dotted curves to the flare state, and the solid curves to the temporal average.

brightness profile of the disk beyond $\sim 35$ AU. Another explanation must be found.

\section{Diffusion of dust particles in the outer disk. II. Wind pressure force}

Another possible way for dust particles to blow into the outer disk is that they interact with a stellar wind. Since AU Mic is a late type star, a solar-like coronal wind is likely to arise from the star. This is supported by the coronal activity of AU Mic.

\subsection{Dynamics of grains in the presence of a wind}

The force due to a flux of protons arising from the star is very similar to the radiation force, and may be described as

$$
\left.m \frac{\mathrm{d}^{2} \boldsymbol{r}}{\mathrm{d} t^{2}}\right|_{\mathrm{SW}}=\Phi_{\mathrm{p}} \sigma \frac{C_{\mathrm{D}}}{2}\left[\left(1-\frac{\dot{r}}{c}\right) \hat{\boldsymbol{u}}-\frac{\boldsymbol{v}}{c}\right]
$$


(Gustafson 1994), where $\Phi_{\mathrm{p}}$ is the momentum density of the proton flux at the considered stellar distance, and $C_{\mathrm{D}}$ is the free molecular drag coefficient. This equation is, of course, very similar to Eq. (16), and as for the radiation pressure case, the velocity independent term $\Phi_{\mathrm{p}} \sigma\left(C_{\mathrm{D}} / 2\right) \hat{\boldsymbol{u}}$ is dominant. Logically, we define the dimensionless ratio $\beta_{\mathrm{sw}}$ of that force to stellar gravity, so that the equation of motion now becomes similar to Eq. (17), with $\beta_{\mathrm{pr}}$ changed to $\beta_{\mathrm{sw}}$. The $\beta_{\mathrm{sw}}$ ratio has been introduced in the solar wind case by Mukai \& Yamamoto (1982) and Gustafson (1994). We write

$\beta_{\mathrm{sw}}=\frac{\Phi_{\mathrm{p}} \sigma\left(C_{\mathrm{D}} / 2\right)}{G M m / r^{2}}$.

The main difference between $\beta_{\mathrm{pr}}$ and $\beta_{\mathrm{sw}}$ is that the latter is not necessarily independent of the stellar distance $r$. We investigate this dependence. If $\rho_{\mathrm{sw}}$ and $v_{\mathrm{sw}}$ are the mass density and velocity of the wind at distance $r$, we have $\Phi_{\mathrm{p}}=\rho_{\mathrm{sw}} v_{\mathrm{sw}}^{2}$. The coefficient $C_{\mathrm{D}}$ is given by Gustafson (1994):

$$
\begin{gathered}
C_{\mathrm{D}}=\frac{2 S^{2}+1}{S^{3} \sqrt{\pi}} \mathrm{e}^{-S^{2}}+\frac{4 S^{4}+4 S^{2}-1}{2 S^{4}} \operatorname{erf}(S) \\
+\frac{2(1-\epsilon) \sqrt{\pi}}{3 S} \sqrt{\frac{T_{\mathrm{d}}}{T_{\mathrm{p}}}}\left(1+Y_{\mathrm{p}} \frac{m_{\mathrm{s}}}{m_{\mathrm{p}}}\right),
\end{gathered}
$$

where $S=\sqrt{m_{\mathrm{p}} /\left(2 k T_{\mathrm{p}}\right)} u$ is the ratio of the relative velocity $u$ to the thermal velocity of the protons $\sqrt{2 k T_{\mathrm{p}} / m_{\mathrm{p}}}(k$ is Boltzmann's constant), $\epsilon$ is the fraction of re-emitted protons at dust temperature $T_{\mathrm{d}}$, and $Y_{\mathrm{p}}$ is a coefficient introduced by Mukai \& Yamamoto (1982) to account for sputtering of molecules of mass $m_{\mathrm{s}}$. In practice, for any standard wind model, at sufficiently large distance we have $u \simeq v_{\text {sw }}$ (we neglect the orbital velocity), and for $S \gg 1, C_{\mathrm{D}}$ is close to 2 .

If we assume spherical symmetry, then we may write the continuity equation $\dot{M}=4 \pi r^{2} \rho_{\text {sw }} v_{\text {sw }}$, where $\dot{M}$ is the mass loss rate. If we assume that the grain is spherical, with radius $s$ and density $\rho$, then the ratio $\beta_{\text {sw }}$ may be rewritten as

$\beta_{\mathrm{sw}}=\frac{3}{32 \pi} \frac{\dot{M} v_{\mathrm{sw}} C_{\mathrm{D}}}{G M \rho s}$.

Thus, $\beta_{\mathrm{sw}}$ may be a function of the distance to the star, as $v_{\mathrm{sw}}$ and $C_{\mathrm{D}}$ are not necessarily constant throughout the disk. The stellar wind blow-out size then reads $\left(\beta_{\mathrm{sw}}=0.5\right)$

$s_{\mathrm{sw}}=\frac{3}{16 \pi} \frac{\dot{M} v_{\mathrm{sw}} C_{\mathrm{D}}}{G M \rho}$ with $C_{\mathrm{D}} \simeq 2$.

\subsection{Wind model and mass loss rate}

If we want to describe further the impact of the wind pressure force on the dust dynamics, we need to adopt a wind model. The most standard model for a solar-like coronal wind is the transonic isothermal solution by Parker (1958). This model has the advantage of being fully controlled by only two parameters, namely the temperature $T_{\mathrm{p}}$ and the mass loss rate $\dot{M}$, or equivalently $T_{\mathrm{p}}$ and the number density $n_{0}$ at the base of the corona. The relation between these parameters is

$\dot{M}=4 \pi R^{2} \frac{n_{0} m_{\mathrm{p}}}{2} \sqrt{\frac{2 k T_{\mathrm{p}}}{m_{\mathrm{p}}}}\left(\frac{\lambda^{2}}{4} \mathrm{e}^{3 / 2-\lambda}\right)$,

where $R$ is the radius of the star and with

$\lambda=\frac{G M / R}{2 k T_{\mathrm{p}} / m_{\mathrm{p}}}$.
The velocity solution of the wind is implicitly given by

$w \mathrm{e}^{-w^{2} / 2}=\frac{\lambda^{2} R^{2}}{4 r^{2}} \exp \left(\frac{3}{2}-\frac{\lambda R}{r}\right)$,

where $w=\sqrt{m_{\mathrm{p}} /\left(2 k T_{\mathrm{p}}\right)} v_{\mathrm{sw}}$ is the velocity normalized to the thermal velocity. With the above relations, we may compute the $\beta_{\mathrm{sw}}$ ratio as a function of the distance $r$ from the star and of the parameters $T_{\mathrm{p}}$ and $n_{0}$. The analytical expression is rather complex and makes use of Lambert's $W$ function that comes from the solution of Eq. (32), but here we are interested in the numerical values of $\beta_{\mathrm{sw}}$.

Our first remark is that contrary to $Q_{\mathrm{pr}}$, the coupling coefficient $C_{\mathrm{D}}$ here is totally independent of the size of the grain. This means that for spherical grains, $\beta_{\mathrm{sw}}$ will be exactly $\propto s^{-1}$ (see Eq. (28)), for any grain size. This behavior was noted by Mukai $\&$ Yamamoto (1982). Hence, for sufficiently small grains, $\beta_{\mathrm{sw}}$ may reach arbitrarily high values. However, Mukai \& Yamamoto (1982) show that in the case of the solar wind characterized by $T_{\mathrm{p}} \simeq 10^{6} \mathrm{~K}$ and $n_{0} \simeq 10^{9} \mathrm{~cm}^{-3}, \beta_{\mathrm{sw}}$ remains several orders of magnitude less than $\beta_{\text {pr }}$ for usual grain sizes ( $\beta_{\mathrm{sw}}$ would reach 0.5 or 1 for radii less than $1 \mathrm{~nm}$ ). As a matter of fact, in the solar environment, the wind pressure is usually compared to PoyntingRobertson drag rather than to the full radiation pressure, with a ratio to PRD reaching $\sim 0.3$ (Gustafson 1994).

The situation may be drastically different with an active star like AU Mic. Theoretical studies have shown that active M-dwarfs may have wind mass loss rates typically reaching a few times $10^{-12} M_{\odot} \mathrm{yr}^{-1}$ (Wargelin \& Drake 2001; Wood et al. 2002), i.e., more than 100 times the solar value. Concerning AU Mic in particular, there are several density and temperature estimates in the coronal plasma near the star that are deduced from the observation and analysis of spectral lines of highly ionized species. Monsignori-Fossi et al. (1996), based on the study of EUV lines of Fe XXI and Fe XXIV, give a temperature $T_{\mathrm{p}} \simeq 8 \times 10^{6} \mathrm{~K}$ in quiescent phases and $2-5 \times 10^{7} \mathrm{~K}$ in flare phases; they also derive electron densities (that we will take equal to $n_{0}$ ) between $3 \times 10^{12}$ and $2 \times 10^{13} \mathrm{~cm}^{-3}$ during flares. Pagano et al. (2000) find $n_{0}=6.3 \times 10^{10} \mathrm{~cm}^{-3}$ in a quiescent state, with some regions with $n_{0}=5 \times 10^{11} \mathrm{~cm}^{-3}$. Completing that study, Robinson et al. (2001) derive $n_{0}=1.7 \times 10^{12} \mathrm{~cm}^{-3}$ as an average for flare phases. We thus decided to assume as typical values of $n_{0}=6.3 \times 10^{10} \mathrm{~cm}^{-3}$ and $T_{\mathrm{p}} \simeq 8 \times 10^{6} \mathrm{~K}$ for quiescent state, and, $n_{0}=1.7 \times 10^{12} \mathrm{~cm}^{-3}$ and $T_{\mathrm{p}} \simeq 3 \times 10^{7} \mathrm{~K}$ during flares. Via Eq. (30), this results in $\dot{M}=9.4 \times 10^{-13} M_{\odot} \mathrm{yr}^{-1}$ in quiescent state and a peak of $\dot{M}=4.9 \times 10^{-11} M_{\odot} \mathrm{yr}^{-1}$ during flares. If we assume that flares are present $10 \%$ of the time, the average mass loss rate is $5.7 \times 10^{-12} M_{\odot} \mathrm{yr}^{-1} \simeq 3 \times 10^{2} \dot{M}_{\odot}$, which is in perfect agreement with the theoretical estimates quoted above. In particular, Wood et al. (2002) derive a power-law relationship between the X-ray activity of dwarfs and their mass loss rate. Applied to AU Mic, this gives $\dot{M} \sim 1-2 \times 10^{-12} M_{\odot} \mathrm{yr}^{-1}$ (Plavchan et al. 2005).

\subsection{Origin of the disk surface brightness profile}

We are now able to compute the $\beta_{\mathrm{sw}}$ ratio for any grain size at any distance from the star. This is done in Fig. 11 , where $\beta_{\mathrm{sw}}$ is plotted as a function of the stellar distance, in quiescent and flare regimes, for a grain of radius $s=0.1 \mu \mathrm{m}$. The calculation is done with $C_{\mathrm{D}}$ computed from Eq. (27) and neglecting sputtering $\left(C_{\mathrm{D}}\right.$ turns out to remain between 2.0 and 2.1 over the whole range of stellar distances considered). The behavior for other grain sizes may be directly deduced from this plot, as we 


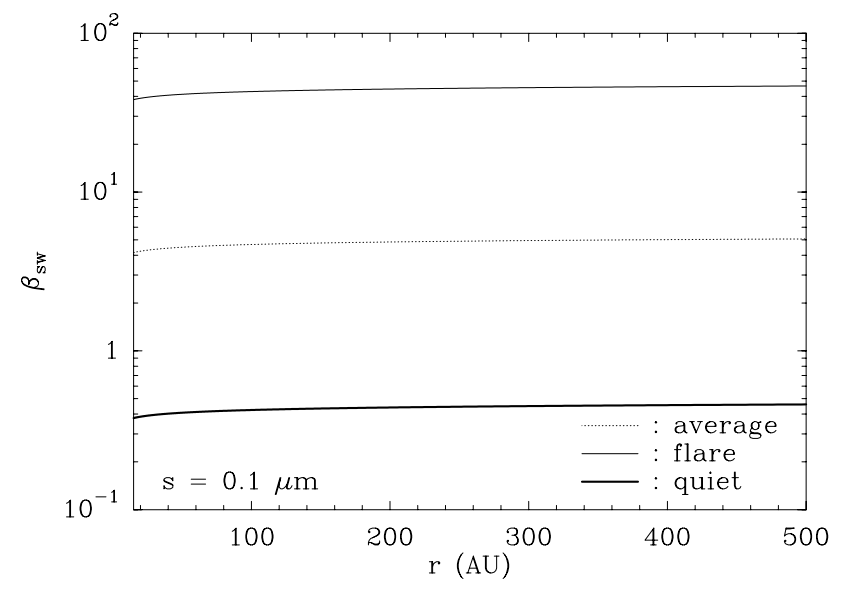

Fig. 11. $\beta_{\mathrm{sw}}$ ratio as a function of the distance to the star, computed for a spherical silicate grain with radius $s=0.1 \mu \mathrm{m}$, in quiescent (thick solid line) and flare (thin solid line) states. The dotted line is the timeaveraged $\beta_{\mathrm{sw}}$, assuming that flares are present $10 \%$ of the time $(\gamma=0.1)$.

strictly have $\beta_{\mathrm{sw}} \propto s^{-1}$. First of all, we note that there is only little variation of $\beta_{\mathrm{sw}}$ over a wide range of distances from the star. In fact, from Eq. (28) we see that the dependence in $r$ of the $\beta_{\text {sw }}$ ratio is entirely contained in the factor $v_{\mathrm{sw}} C_{\mathrm{D}} . C_{\mathrm{D}}$ remains very close to 2 , and $v_{\mathrm{sw}}$ is a moderately increasing function of $r . \beta_{\mathrm{sw}}$ is thus an increasing function of $r$, but the relative increase is only $12.4 \%$ between $r=15 \mathrm{AU}$ and $r=100 \mathrm{AU}$, and $8.4 \%$ between $r=100 \mathrm{AU}$ and $r=500 \mathrm{AU}$. Hence, considering $\beta_{\mathrm{sw}}$ as independent of $r$, like $\beta_{\mathrm{pr}}$, is an acceptable approximation. This justifies the introduction of the $\beta_{\mathrm{sw}}$ ratio, and shows that the dynamics of particles subject to wind pressure will be somewhat similar to that of particles subject to radiation pressure. In particular, we expect that grains will be pushed away in the outer disk as soon as $\beta_{\text {sw }}$ approaches 0.5 .

Figure 11 shows that such large values of $\beta_{\mathrm{sw}}$ can easily be reached. For $s=0.1 \mu \mathrm{m}$, even in quiescent phase, $\beta_{\mathrm{sw}} \simeq 0.4$, while the exact value depends on the assumed mass loss rate. However, because of the recurrent flare episodes, we must consider the temporal average of $\beta_{\text {sw }}$. This is plotted in Fig. 11 as a dotted line, assuming that flares are present $10 \%$ of the time. Due to flares, grains with $s=0.1 \mu \mathrm{m}$ are actually blown away by the stellar wind. As $\beta_{\mathrm{sw}} \propto s^{-1}$, it turns out that all grains with $s \lesssim 1 \mu \mathrm{m}$ are able to diffuse into the outer disk, and even be ejected from the system. Actually the exact behavior at large distances may depend on the location of the heliopause of the stellar wind, but this is not expected closer to a few hundreds of AU.

The fact that $\beta_{\mathrm{sw}}$ is almost constant over several orders of magnitude of stellar distances, and that $\beta_{\mathrm{sw}}$ is able to reach sufficiently large values, shows that particles subject to wind pressure will behave almost as if they were subject to a comparable radiation pressure. This explains why the surface brightness profile along the disk midplane $\left(\propto r^{-4 \cdots-5}\right)$ resembles that of the $\beta$ Pictoris disk, the latter being due to the effects of the intense radiation pressure (Lecavelier des Étangs et al. 1996; Augereau et al. 2001; Thébault \& Augereau 2005).

From this study, we conclude that in the AU Mic system, the stellar wind pressure is expected to play almost the same role as the radiation pressure in other debris disks around earlier type stars like $\beta$ Pictoris. Interestingly, Roberge et al. (2005) also came to the conclusion that the short lifetime of the circumstellar gas in the AU Mic system can only be explained by dissipation mechanisms other than photo-evaporation, including a possible stellar wind. Plavchan et al. (2005) also proposed that the dynamics of the grains in the inner AU Mic system could be controlled by the drag component of the stellar wind. Given that the collision time scale we find within $\sim 30$ AU compares to the stellar wind drag time scale inferred by Plavchan et al. (2005) (time scale rescaled, assuming $\dot{M} \simeq 3 \times 10^{2} \dot{M}_{\odot}$ ), the drag force may play a significant role in explaining the disk clearing in the inner regions. Interestingly, we find that the role of the flares, neglected in previous studies, is significant.

\section{Summary and conclusion}

We have calculated the surface density profiles of the edge-on debris disk around the M-type star AU Mic by performing a direct inversion of two brightness profiles: the $H$-band profiles obtained by Liu (2004) with the Keck adaptive optics system and the HST/ACS $V$-band profiles from Krist et al. (2005). We have solved the integral equation that links the scattered light surface brightness profile along the disk midplane to the disk surface density. The solution to this equation only depends on the light scattering phase function characterized by the asymmetry parameter $|g|$, which is constrained to be smaller than 0.6. From the analysis of the $H$-band observations, we find that the dust distribution peaks around $35 \mathrm{AU}$, close to the location of the power law breakup in the surface brightness profile. The surface density profiles inferred from the inversion of the visible images are quite different, and this difference may partly come from dust properties varying throughout the disk midplane. Even if the exact azimuthal profile of the disk cannot be unambiguously derived, the disk turns out to be an obviously non-symmetrical ring, as the two NE and SW branches are not identical.

From the analysis of the disk colors measured in the visible with the HST/ACS instrument (Krist et al. 2005), we constrain the grain size distribution to be steeper than the classical $s^{-3.5}$ differential size distribution. Grains as small as $0.1 \mu \mathrm{m}$ in radius are, in addition, required to explain the observations when astronomical silicates are assumed. We infer a total mass of silicates grains smaller than $1 \mathrm{~mm}$ equal to $3.5-8 \times 10^{-4} M_{\oplus}$. This dust mass compares the mass of the Ceres asteroid in the Solar System, but it is 14 to 30 times smaller than the dust mass inferred from millimeter observations by Liu et al. (2004). It is also 9 to 20 times smaller than the mass we infer from the disk SED fitting. This difference cannot be explained by the disk optical thickness since the disk is optically thin in all directions and at all wavelengths $\left(\tau_{\|}<0.04\right.$ at visible wavelengths). Possible explanations for this discrepancy include the fact that the simple power law size distribution that we find from the interpretation of the visible observations can probably not be extrapolated all the way to millimeter grain sizes (see, e.g., the discussion in Thébault et al. 2003). It may also be possible that assuming hard spheres to calculate the grain optical properties is not appropriate if the grains have complex structures.

We then explored whether the similarities between the surface brightness profiles of the AU Mic and $\beta$ Pictoris disks are coincidental, or if they could result from similar dynamical processes. The global properties of the dust distribution in the $\beta$ Pictoris disk are very well explained by the role of radiation pressure. In this context, the breakup in surface brightness (observed at $120 \mathrm{AU}$ ) corresponds to the outer edge of the planetesimal disk that produces the dust particles (Augereau et al. 2001). A very similar picture may apply to the AU Mic disk, but at a distance of $\sim 35 \mathrm{AU}$. The main difference lies in the dust diffusion mechanism. The radiation pressure from AU Mic appears to be an inefficient means of diffusing dust into the outer 
disk, even when the X-ray and UV flares are taken into account. Conversely, the corpuscular pressure from a stellar wind is very likely to be much more efficient. In fact, in the AU Mic environment, the wind pressure turns out to play an almost identical role as the radiation pressure in the $\beta$ Pictoris disk, with, in both cases, a constant (or almost constant) ratio to stellar gravity. This explains why the surface brightness profiles of the two disks are similar, despite different generating mechanisms.

This comparison between debris disks around early and latetype stars would be generic if the additional role of flares were not significant in the case of AU Mic. The flares contribute to considerably enhance the effect of the stellar wind, and consequently to transport many additional particles in the outer disk that would otherwise remain close to their parent bodies. Debris disks may be common around late-type stars, but an intense and frequent coronal activity may help to make them sufficiently extended to be detectable.

Acknowledgements. We thank M. Liu, P. Thébault, the FOST (Grenoble), and the AstroChem (Leiden) team members, as well as the referee, P. Kalas, for helpful comments and suggestions. This work was partly supported by the European Community's Human Potential Programme under contract HPRN-CT2002-00308, PLANETS.

\section{References}

Artymowicz P. 1988, ApJ, 335, L79

Augereau, J. C., Lagrange, A. M., Mouillet, D., Papaloizou, J. C. B., \& Grorod, P. A. 1999, A\&A, 348, 557

Augereau, J. C., Nelson, R. P., Lagrange, A. M., Papaloizou, J. C. B., \& Mouillet, D. 2001, A\&A, 370, 447

Backman, D., \& Paresce, F. 1993, in Protostars and Planets III, ed. E. H. Levy and Lunine (Tucson: University of Arizona Press), 1253

Backman, D. E., Dasgupta, A., \& Stencel, R. E. 1995, ApJ, 450, L35

Barrado y Navascués, D., Stauffer, J. R., Song, I., \& Caillault, J.-P. 1999, ApJ, 520, L123

Beust H., Karmann C., \& Lagrange A.-M. 2001, A\&A, 366, 945

Brandeker, A., Liseau, R., Olofsson, G., \& Fridlund, M. 2004, A\&A, 413, 681

Burns, J. A., Lamy, P. L., \& Soter, S. 1979, Icarus, 40, 1

Chen, C. H., Patten, B. M., Werner, M. W., et al. 2005, ApJ, 634, 1372

Cully, S. L., Siegmund, O. H. W., Vedder, P. W., \& Vallerga, J. V. 1993, ApJ, 414, L49

Dohnanyi, J. W. 1969, J. Geophys. Res., 74, 2531
Fitzgerald, M., Graham, J., Kalas, P., \& Matthews, B. 2004, American Astronomical Society Meeting Abstracts, 205, 29.07

Golimowski, D. A., Ardila, D. R. Krist, J. E., et al. 2006, AJ, 131, 3109

Gustafson, B. Å. S 1994, Ann. Rev. Earth Planet. Sci., 22, 553

Hauschildt, P. H., Allard, F., \& Baron, E. 1999, ApJ, 512, 377

Henyey, L. G., \& Greenstein, J. L. 1941, ApJ, 93, 70

Houdebine, E. R., \& Doyle, J. G. 1994, A\&A, 289, 185

Hünsch, M., Schmitt, J. H. M. M., Sterzik, M. F., \& Voges, W. 1999, A\&AS, 135,319

Kalas, P., Liu, M. C., \& Matthews, B. C. 2004, Science, 303, 1990

Krist, J. E., Ardila, D. R., Golimowski, D. A., et al. 2005, AJ, 129, 1008

Kunkel, W. 1973, ApJS, 430, 342

Landsman, W., \& Simon, T., 1993, ApJ, 408, 305

Laor, A., \& Draine, B. T. 1993, ApJ, 402, 441

Lecavelier Des Étangs, A., Vidal-Madjar, A., \& Ferlet, R. 1996b, A\&A, 307, 542

Liu, M. C. 2004, Science, 305, 1442

Liu, M. C., Matthews, B. C., Williams, J. P., \& Kalas, P. G. 2004, ApJ, 608, 526

Magee, H. R. M., Güdel, M., Audard, M., \& Mewe, R. 2003, Adv. Space Res., 32, 1149

Monsignori-Fossi, B. C., \& Landini, M. 1994, A\&A, 284, 900

Monsignori-Fossi, B. C., Landini, M., Del Zanna, G., \& Bowyer, S. 1996, ApJ, 466,427

Mouillet, D., Larwood, J. D., Papaloizou, J. C. B., \& Lagrange, A. M. 1997, MNRAS, 292, 896

Metchev, S. A., Eisner, J. A., Hillenbrand, L. A., \& Wolf, S. 2005, ApJ, 622, 451

Mitra-Kraev, U., Harra, L. K., Güdel, M., et al. 2005, A\&A, 431, 679

Mukai, T., \& Yamamoto, T. 1982, A\&A, 107, 97

Najita, J., \& Williams, J. P. 2005, ApJ, 635, 625

Nakano, T. 1990, ApJ, 355, L43

Pagano, I., Linsky, J. L., Carkner, L., et al. 2000, ApJ, 532, 497

Pantin, E., Lagage, P. O., \& Artymowicz, P. 1997, A\&A, 327, 1123

Parker, E., 1958, ApJ, 128, 664

Plavchan, P., Jura, M., \& Lipscy, S. J. 2005, ApJ, 631, 1161

Roberge, A., Weinberger, A. J., Redfield, S., \& Feldman, P. D. 2005, ApJ, 626, L105

Robinson, R. D., Linsky, J. L., Woodgate, B. E., \& Timothy, J. G. 2001, ApJ, 554,368

Roques, F., Scholl, H., Sicardy, B., \& Smith, B. A. 1994, Icarus, 108, 37

Schneider, G., et al. 2006, ApJ, in press [arXiv:astro-ph/0606213]

Telesco, C. M., Fisher, R., Scott, Wyatt, M. C., et al. 2005, Nature, 433, 133

Thébault, P., Augereau, J. C., \& Beust, H. 2003, A\&A, 408, 775

Thébault, P., \& Augereau, J.-C. 2005, A\&A, 437, 141

Tsikoudi V., \& Kellett B. J. 2000, MNRAS, 319, 1147

Weingartner, J. C., \& Draine, B. T. 2001, ApJ, 548, 296

Wargelin, B. J., \& Drake, J. J. 2001, ApJ, 546, L57

Wood, B. E., Müller, H.-R., Zank, G. P., \& Linsky, J. L. 2002, ApJ, 574, 412

Wyatt, M. C. 2005, A\&A, 433, 1007

Zuckerman, B., Song, I., Bessell, M. S., \& Webb, R. A. 2001, ApJ, 562, L87 


\section{Online Material}




\section{Appendix A: Numerical scheme to invert Eq. (5)}

The product integration method is a simple, but efficient and generally numerically stable technique that can be used to invert an integral equation. This appendix describes the main steps towards the implementation of this method in order to solve Eq. (5). We refer to Sects. 2 and 3.3 for the notations used in this appendix.

We expand Eq. (5) as a sum of $n$ integrals over ranges of equal size $h$

$S(y)=\sum_{j=0}^{n-1} \int_{r_{\max }-(j+1) h}^{r_{\max }-j h} \sigma_{\text {sca }} \Sigma_{0}(r) \frac{A(r, y)}{r \sqrt{r^{2}-y^{2}}} \mathrm{~d} r$,

where $y=r_{\max }-n h$ and

$A(r, y)=\Phi^{*} D_{z}^{-1}(r, \theta) \Theta(r, \theta)(f(\theta)+f(\pi-\theta))$.

We note $r_{j}=r_{\max }-j h$, which gives $r_{n}=y$ and $r_{0}=r_{\max } . S(y)$ and $A(r, y)$ are supposed to be two known functions of $r$ and/or $y$.

We assume that for $r$ within the range $r_{j+1} \leq r \leq r_{j}$, $\sigma_{\text {sca }} \Sigma_{0}(r) A(r, y)$ can be approximated, to first order, by its value at the middle of the interval of size $h$ (product integral method), namely at the position $r=r_{j+\frac{1}{2}}$. The smaller $h$ is, the better the approximation is. The surface brightness profile $S(y)=S\left(r_{n}\right)$ thus reads

$S\left(r_{n}\right) \simeq \sum_{j=0}^{n-1} \sigma_{\mathrm{sca}} \Sigma_{0}\left(r_{j+\frac{1}{2}}\right) A\left(r_{j+\frac{1}{2}}, r_{n}\right) I\left(r_{j+1}, r_{j}, r_{n}\right)$,

where $I\left(r_{j+1}, r_{j}, r_{n}\right)=\int_{r_{j+1}}^{r_{j}} \frac{1}{r \sqrt{r^{2}-r_{n}^{2}}} \mathrm{~d} r$

and $\int \frac{1}{r \sqrt{r^{2}-r_{n}^{2}}} \mathrm{~d} r=-\frac{1}{r_{n}} \arctan \left(\frac{r_{n}}{\sqrt{r^{2}-r_{n}^{2}}}\right)$.

Since $h$ is small compared to the size of the disk, taking $n=1$ in the above expression of $S\left(r_{n}\right)$ leads to a first crude estimate of $\sigma_{\text {sca }} \Sigma_{0}(r)$ close the outer edge $r_{\max }$ of the disk

$\sigma_{\text {sca }} \Sigma_{0}\left(r_{\frac{1}{2}}\right) \simeq \frac{S\left(r_{1}\right)}{A\left(r_{\frac{1}{2}}, r_{n}\right) I\left(r_{1}, r_{0}, r_{n}\right)}$.

For $n \geq 2$, we can write

$$
\begin{aligned}
S\left(r_{n}\right) \simeq & \sum_{j=0}^{n-2} \sigma_{\mathrm{sca}} \Sigma_{0}\left(r_{j+\frac{1}{2}}\right) A\left(r_{j+\frac{1}{2}}, r_{n}\right) I\left(r_{j+1}, r_{j}, r_{n}\right) \\
& +\sigma_{\mathrm{sca}} \Sigma_{0}\left(r_{n-\frac{1}{2}}\right) A\left(r_{n-\frac{1}{2}}, r_{n}\right) I\left(r_{n}, r_{n-1}, r_{n}\right)
\end{aligned}
$$

and then

$$
\begin{aligned}
\sigma_{\mathrm{sca}} \Sigma_{0}\left(r_{n-\frac{1}{2}}\right) \simeq & \frac{S\left(r_{n}\right)}{A\left(r_{n-\frac{1}{2}}, r_{n}\right) I\left(r_{n}, r_{n-1}, r_{n}\right)} \\
& -\frac{\sum_{j=0}^{n-2} \sigma_{\mathrm{sca}} \Sigma_{0}\left(r_{j+\frac{1}{2}}\right) A\left(r_{j+\frac{1}{2}}, r_{n}\right) I\left(r_{j+1}, r_{j}, r_{n}\right)}{A\left(r_{n-\frac{1}{2}}, r_{n}\right) I\left(r_{n}, r_{n-1}, r_{n}\right)} .
\end{aligned}
$$

With this simple algorithm, we can directly invert Eq. (5) to get the product of the surface number density $\Sigma_{0}(r)$ by the scattering cross section $\sigma_{\text {sca }}$ as a function of the distance to the star. $\sigma_{\text {sca }} \Sigma_{0}$ is reconstructed from the outer disk edge $\left(r_{\max }\right)$ down to the closest possible distance. At each distance $r$ from the star, $\sigma_{\text {sca }} \Sigma_{0}(r)$ is thus calculated using the values of $\sigma_{\text {sca }} \Sigma_{0}$ previously estimated at distances larger than $r$. We checked that with this simple algorithm we could reproduce the analytic results obtained by Nakano (1990) for an edge-on disk with a decreasing power-law surface brightness profile.

\section{Appendix B: Accounting for non-axisymmetric disks: the $\Theta(r, \theta)$ smoothing function}

The inversion of the NW and SE surface brightness profiles do not depend on the position angle on the skyplane at which they have been measured. To simplify the discussion, and as far as the inversion is concerned, we will thus always assume that the brightness profile is measured along the $y$-axis, and that $y>0$ (see Fig. 1).

If $h$ (as defined in Appendix A) could be infinitely small, the inversion of Eq. (5) would lead to the reconstruction of the surface density of the disk precisely along the $y$-axis, namely at a polar angle $\theta=\pi / 2$. However, because $\sigma_{\text {sca }} \Sigma_{0}(r)$ is estimated at the middle of an interval of finite size $h$, the surface density is calculated at polar angles $\theta_{0}$ that slightly deviate from $\pi / 2$ and with $\lim \theta_{0}=\pi / 2$ when $h \rightarrow 0$. The smoothing function $\Theta(r, \theta)$ introduced in Sect. 3.3 to account for non-axisymmetric disks reads

$\Theta_{\mathrm{SE}}(r, \theta)=\frac{1-p}{2} \times\left(\frac{\theta}{\theta_{0}}\right)^{\delta}+\frac{1+p}{2}$

for the SE side of the disk, and

$\Theta_{\mathrm{NW}}(r, \theta)=\frac{1-p^{-1}}{2} \times\left(\frac{\theta}{\theta_{0}}\right)^{\delta}+\frac{1+p^{-1}}{2}$

for the NW side, with $0 \leq \theta \leq \pi / 2$ and where $p$ is given by

$p=\left[\sigma_{\mathrm{sca}} \Sigma_{0}(r)\right]_{\mathrm{NW}} /\left[\sigma_{\mathrm{sca}} \Sigma_{0}(r)\right]_{\mathrm{SE}}$.

\section{Appendix C: Dynamics of particles with time-variable radiation pressure}

We investigate here the dynamics of particles with a timevariable $\beta_{\mathrm{pr}}$. We assume that the star periodically alternates quiescent and flare phases, respectively characterized for a given grain by $\beta_{\mathrm{pr}, \mathrm{q}}$ and $\beta_{\mathrm{pr}, \mathrm{f}}$ ratios, with $\beta_{\mathrm{pr}, \mathrm{f}}>\beta_{\mathrm{pr}, \mathrm{q}}$. The star is assumed to spend a fixed fraction $\gamma$ of the time in flare phases. Strictly speaking, the grain follows bits of Keplerian orbits alternatively characterized by dynamical masses $M\left(1-\beta_{\mathrm{pr}, \mathrm{q}}\right)$ and $M\left(1-\beta_{\mathrm{pr}, \mathrm{f}}\right)$. What is the global effect? First, we should note that this problem cannot be treated by a classical perturbation method typical of celestial mechanics, as the "perturbation" (the flares effect) overcomes the main Keplerian forces.

Let us consider a simple episode, characterized by a first quiescent phase $\left(\beta_{\mathrm{pr}}=\beta_{\mathrm{pr}, \mathrm{q}}\right)$, a flare $\left(\beta_{\mathrm{pr}}=\beta_{\mathrm{pr}, \mathrm{f}}\right)$, and a second quiescent phase $\left(\beta_{\mathrm{pr}}=\beta_{\mathrm{pr}, \mathrm{q}}\right)$. During each of these phases, the grain follows a different Keplerian orbit. Due to the flare, the Keplerian orbit during the first quiescent phase differs from that during the second quiescent phase. We now would like to compute the orbital change.

First, we note that during the whole episode, the angular momentum remains unchanged. Even if $\beta_{\mathrm{pr}}$ is time-variable, the resulting acceleration keeps pointing towards the central star, keeping the angular momentum constant. Hence, the orbital plane of the motion remains unchanged, as does the quantity $a\left(1-e^{2}\right)$ during the successive quiescent phases. Second, the orbital energy $\delta E$ change between the two quiescent phases may be computed easily, using the continuity of the velocity during the whole episode. Using a simple algebra, we derive

$\delta E=G M\left(\beta_{\mathrm{pr}, \mathrm{f}}-\beta_{\mathrm{pr}, \mathrm{q}}\right) \frac{r_{2}-r_{1}}{r_{1} r_{2}}$

where $r_{1}$ and $r_{2}$ are the distances of the particle to the star at the beginning of the flare and at the end, respectively. Now, the 
important remark is that the period of recurrence of the flares (a few days at most) is far less than the orbital period of the particle (hundreds of years at 35-40 AU). The distance change during the whole episode may be regarded as infinitesimal.

Let us now consider the time span between the beginning of a flare and the beginning of the next flare. During this time span, we first have a flare that generates a (quiescent) orbital energy change described by Eq. (C.1), and a quiescent phase that keeps it constant up to the next flare. The time span considered may be regarded as infinitesimal compared to the orbital period, so that the motion of the particle during this phase is nearly uniform. If $\mathrm{d} r$ is the infinitesimal distance change during this phase, we should have $r_{2}-r_{1} \simeq \gamma \mathrm{d} r$ in Eq. (C.1). The orbital energy change within $\mathrm{d} r$ then is

$\mathrm{d} E=G M\left(\beta_{\mathrm{pr}, \mathrm{f}}-\beta_{\mathrm{pr}, \mathrm{q}}\right) \frac{\gamma \mathrm{d} r}{r^{2}}$.

Integrating this equation, we derive the macroscopic energy change that corresponds to the stellar distance change from $r$ to $r^{\prime}$

$\Delta E=G M \gamma\left(\beta_{\mathrm{pr}, \mathrm{f}}-\beta_{\mathrm{pr}, \mathrm{q}}\right)\left(\frac{1}{r}-\frac{1}{r^{\prime}}\right)$.

This is equivalent to saying that the quantity

$E_{\mathrm{eff}}=E+\frac{G M \gamma\left(\beta_{\mathrm{pr}, \mathrm{f}}-\beta_{\mathrm{pr}, \mathrm{q}}\right)}{r}$.
Given the expression of the energy $E$, we derive

$$
\begin{aligned}
E_{\mathrm{eff}} & =\frac{1}{2} v^{2}-\frac{G M\left(1-\beta_{\mathrm{pr}, \mathrm{q}}\right)}{r}+\frac{G M \gamma\left(\beta_{\mathrm{pr}, \mathrm{f}}-\beta_{\mathrm{pr}, \mathrm{q}}\right)}{r} \\
& =\frac{1}{2} v^{2}-\frac{G M\left(1-\beta_{\mathrm{pr}, \mathrm{eff}}\right)}{r}
\end{aligned}
$$

where

$\beta_{\mathrm{pr}, \mathrm{eff}}=(1-\gamma) \beta_{\mathrm{pr}, \mathrm{q}}+\gamma \beta_{\mathrm{pr}, \mathrm{f}}$.

$E_{\text {eff }}$ is just the energy corresponding to a Keplerian orbit with central mass $M\left(1-\beta_{\text {pr,eff }}\right)$, i.e., to a particle feeling a $\beta_{\mathrm{pr}}=\beta_{\mathrm{pr}, \mathrm{eff}}$ ratio from the star. Combined to the preservation of angular momentum, we see that, on average, the dust particle will keep following a Keplerian orbit around the star, but will be characterized by a $\beta_{\text {pr }}=\beta_{\text {preff }}$ ratio. We checked this result numerically with a dedicated integrator.

$\beta_{\mathrm{pr}, \mathrm{eff}}$ is only the temporal average of the $\beta_{\mathrm{pr}}$ ratio between flare and quiescent phases. Finally, the net result is that whenever the radiation pressure is time-variable, the grain behaves as if it was feeling a $\beta_{\text {pr }}$ ratio from the star that is just the temporal average of the variable $\beta_{\mathrm{pr}}$. We should nevertheless not forget that this is valid if the recurrence frequency of the flares largely overcomes the orbital frequency of the orbit. Significantly different outcomes should be expected for instance if there were a resonance between the mean motion of the orbit and the recurrence frequency of flares, which is not the case here. 